\title{
Ergodicity for the stochastic Complex Ginzburg-Landau equations
}

\author{
Cyril Odasso ${ }^{\mathrm{a}, \mathrm{b}}$ \\ a Ecole Normale Supérieure de Cachan, Antenne de Bretagne, avenue Robert Schuman, campus de Ker Lann, 35170 Bruz, France \\ b IRMAR, UMR 6625 du CNRS, campus de Beaulieu, 35042 Rennes cedex, France
}

Received 7 June 2004; received in revised form 12 May 2005; accepted 16 June 2005

Available online 7 December 2005

\begin{abstract}
We study a stochastic Complex Ginzburg-Landau (CGL) equation driven by a smooth noise in space and we establish exponential convergence of the Markov transition semi-group toward a unique invariant probability measure. Since Doob Theorem does not seem not to be useful in our situation, a coupling method is used. In order to make this method easier to understand, we first focus on two simple examples which contain most of the arguments and the essential difficulties.
\end{abstract}

(c) 2005 Elsevier SAS. All rights reserved.

\section{Résumé}

Nous considérons l'équation de Ginzburg-Landau complexe bruitée par un bruit blanc en temps et régulier par rapport aux variables spatiales et nous établissons le caractère exponentiellement mélangeant du semi-groupe de Markov vers une unique mesure de probabilité invariante. Comme le Théorème de Doob semble ne pas pouvoir être appliqué, nous utilisons une méthode de couplage. Pour une meilleur compréhension, nous focaliserons d'abord notre attention sur deux exemples qui bien que très simples contiennent l'essentiel des difficultés.

@ 2005 Elsevier SAS. All rights reserved.

MSC: 35Q60; 37H99; 37L99; 60H10; 60H15

Keywords: Stochastic Complex Ginzburg-Landau equations; Markovian transition semigroup; Invariant measure; Ergodicity; Coupling method;

Girsanov's formula; Foias-Prodi estimate

\section{Introduction}

Originally introduced to describe a phase transition in superconductivity [8], the Complex Ginzburg-Landau (CGL) equation also models the propagation of dispersive non-linear waves in various areas of physics such as hydrodynamics $[19,20]$, optics, plasma physics, chemical reaction [10] ...

When working in non-homogenous or random media, a noise is often introduced and the stochastic CGL equation may be more representative than the deterministic one.

The CGL equation arises in the same areas of physics as the non-linear Schrödinger (NLS) equation. In fact, the CGL equation is obtained by adding two viscous terms to the NLS equation. The inviscid limits of the deterministic

E-mail address: cyril_odasso@hotmail.com (C. Odasso). 
and stochastic CGL equation to the NLS equation are established in [2] and [16], respectively. The stochastic NLS equation is studied in [5] and [6].

Ergodicity of the stochastic CGL equation is established in [1] when the noise is invertible and in [9] for the one-dimensional cubic case when the noise is diagonal, does not depend on the solution and is smooth in space.

Our aim in this article is to study ergodicity for stochastic CGL equation under very general assumptions.

Let us recall that the stochastic CGL equation has the form

$$
\begin{cases}\frac{\mathrm{d} u}{\mathrm{~d} t}-(\varepsilon+\mathrm{i}) \Delta u+(\eta+\lambda \mathrm{i})|u|^{2 \sigma} u=b(u) \frac{\mathrm{d} W}{\mathrm{~d} t}, & \\ u(t, x)=0, & \text { for } x \in \delta D, t>0, \\ u(0, x)=u_{0}(x), & \text { for } x \in D .\end{cases}
$$

The unknown $u$ is a complex valued process depending on $x \in D, D \subset \mathbb{R}^{d}$ a bounded domain, and $t \geqslant 0$.

We want to consider noises which may be degenerate and our work is in the spirit of $[3,7,9,12-15,17]$ and [22]. Many ideas of this article are taken from these works. However, we develop several generalisations.

The main idea is to compensate the degeneracy of the noise on some subspaces by dissipativity arguments, the so-called Foias-Prodi estimates. A coupling method is developed in a sufficiently general framework to be applied and prove exponential convergence to equilibrium.

To describe the ideas, it is convenient to introduce $\left(e_{k}\right)_{k \in \mathbb{N}^{*}}$ the eigenbasis of the operator $-\Delta$ with Dirichlet boundary conditions (if periodic boundary conditions were considered, it would be the Fourier basis) and $P_{N}$ the eigenprojector on the first $N$ modes.

The main assumption of the papers cited above as well as in this work is that the noise is non-degenerate on the space spanned by $\left(e_{k}\right)_{1 \leqslant k \leqslant N}$ for $N$ sufficiently large. In [9,15] and [22], the noise is also additive, i.e. $b(u)$ does not depend on $u$. The method developed in [17] allows to treat more general noises and, in [17], $b$ is allowed to depend on $P_{N} u$. However, in this latter work, the author restricts his attention to the case when the high modes are not perturbed by noise. It is claimed that the method can be generalised to treat a noise which hits all components. Such a generalisation is contained in [18] in the purely additive case.

Here we develop also such a generalisation and treat a noise which may hit all modes but depends only on $P_{N} u$. We have chosen to use ideas both from [17] and from [15,22]. We hope that this makes our proof easier to understand. Moreover, we get rid of the assumption that $b$ is diagonal in the basis $\left(e_{k}\right)_{k \in \mathbb{N}^{*}}$.

Also, if we work in the space $L^{2}(D)$, it is not difficult to get a Lyapounov structure and Foias-Prodi estimates. Thus, with an additive noise or with a noise as in [17], our results would be a rather easy applications of these methods.

However, this works only for small values of $\sigma$, namely $\sigma<\frac{2}{d}$. It is well known that the CGL equations are also well-posed for $\sigma \in\left[\frac{2}{d}, \frac{2}{d-2}\right)\left(\sigma \in\left[\frac{2}{d}, \infty\right)\right.$ for $\left.d \in\{1,2\}\right)$ provided we work with $H^{1}(D)$-valued solutions and the nonlinearity is defocussing $(\lambda=1)$. We also develop the coupling method in that context and show that it is possible to find a convenient Lyapounov structure and derive Foias-Prodi estimates. Thus we prove exponential convergence to equilibrium for the noises described above in all the cases when it is known that there exists a unique global solution and an invariant measure.

Moreover, using the smoothing effect of CGL and an interpolation argument, we are able to prove exponential convergence in the Wasserstein norm in $H^{s}(D)$ for any $s<2$. This give convergence to equilibrium for less regular functional.

In order to make the understanding of the method easier, we start with two simple examples which motivate and introduce all arguments in a simpler context. The first example is particularly simple. It introduces the idea of coupling and the use of Girsanov transform to construct a coupling. The second example is similar to the one considered in [17]. However, it contains further difficulties and more details are given. We have tried to isolate every key argument. This is also the opportunity to state a very general result giving conditions implying exponential mixing (Theorem 1.8). It is a strong generalisation of Theorem 3.1 of [15].

Then, in Section 2 we deal with CGL equations. We state and prove the general ergodicity result described above

\section{Preliminary results}

The proof of our result is obtained by the combination of two main ideas: the coupling and the Foias-Prodi estimate. The first subsection is a simple example devoted to understand the use of the notion of coupling. The second subsection 
is a two-dimensional example devoted to understand how we use the two main ideas. The third subsection is the statement of an abstract result which is both fundamental and technical. The other subsections are devoted to the proof of this abstract result. The understanding of the proof of the abstract result is not necessary to the understanding of the rest of the article. On the contrary the three first subsections contain the main ideas of this article.

\subsection{A simple example}

In this subsection we introduce the notion of coupling and we motivate it on a simple example.

Let $\Pi$ the one-dimensional torus. We consider the following example. We denote by $X\left(\cdot, x_{0}\right)$ the unique solution in $\Pi$ of

$$
\frac{\mathrm{d} X}{\mathrm{~d} t}+f(X)=\frac{\mathrm{d} W}{\mathrm{~d} t}, \quad X\left(0, x_{0}\right)=x_{0},
$$

where $f: \Pi \rightarrow \mathbb{R}$ is a Lipschitz function and $W$ is a one-dimensional Brownian motion. It is easy to prove that $X$ is a Markovian process. We denote by $\left(\mathcal{P}_{t}\right)_{t}$ its Markovian transition semigroup.

We recall the definition of $\|\mu\|_{\mathrm{var}}$, the total variation of a finite real measure $\mu$ :

$$
\|\mu\|_{\mathrm{var}}=\sup \{|\mu(\Gamma)| \mid \Gamma \in \mathcal{B}(\Pi)\},
$$

where we denote by $\mathcal{B}(\Pi)$ the set of the Borelian subsets of $\Pi$. It is well known that $\|\cdot\|_{\text {var }}$ is the dual norm of $|\cdot|_{\infty}$. We prove that there exists a unique invariant measure $\nu$ and that for any probability measure $\mu$

$$
\left\|\mathcal{P}_{t}^{*} \mu-v\right\|_{\mathrm{var}} \leqslant c \mathrm{e}^{-\beta t} .
$$

Using a completeness argument and the Markovian property of $X$, we obtain that it is sufficient to prove that for any $\psi: \Pi \rightarrow \mathbb{R}$ Borelian bounded and for any $\left(t, x_{1}, x_{2}\right) \in \mathbb{R}^{+} \times \Pi^{2}$, we have

$$
\left|\mathbb{E} \psi\left(X\left(t, x_{1}\right)\right)-\mathbb{E} \psi\left(X\left(t, x_{2}\right)\right)\right| \leqslant c|\psi|_{\infty} \mathrm{e}^{-\beta t} .
$$

Clearly it is sufficient to find $\left(X_{1}(t), X_{2}(t)\right)$ such that for any $(i, t) \in\{1,2\} \times \mathbb{R}^{+}$, we have $\mathcal{D}\left(X_{i}(t)\right)=\mathcal{D}\left(X\left(t, x_{i}\right)\right)$, where $\mathcal{D}$ means distribution, and

$$
\left|\mathbb{E} \psi\left(X_{1}(t)\right)-\mathbb{E} \psi\left(X_{2}(t)\right)\right| \leqslant c|\psi|_{\infty} \mathrm{e}^{-\beta t} .
$$

Now we introduce the notion of coupling. Let $\left(\mu_{1}, \mu_{2}\right)$ be two distributions on a same space $(E, \mathcal{E})$. Let $(\Omega, \mathcal{F}, \mathbb{P})$ be a probability space and let $\left(Z_{1}, Z_{2}\right)$ be two random variables $(\Omega, \mathcal{F}) \rightarrow(E, \mathcal{E})$. We say that $\left(Z_{1}, Z_{2}\right)$ is a coupling of $\left(\mu_{1}, \mu_{2}\right)$ if $\mu_{i}=\mathcal{D}\left(Z_{i}\right)$ for $i=1,2$.

Remark 1.1. Although the marginal laws of $\left(Z_{1}, Z_{2}\right)$ are imposed, we have a lot of freedom when choosing the law of the couple $\left(Z_{1}, Z_{2}\right)$. For instance, let us consider $\left(W_{1}, W_{2}\right)$ a two-dimensional Brownian motion. Let $\mu$ be the Wiener measure on $\mathbb{R}$, which means that $\mu=\mathcal{D}\left(W_{1}\right)=\mathcal{D}\left(W_{2}\right)$. Then $\left(W_{1}, W_{2}\right),\left(W_{1}^{\prime}, W_{2}^{\prime}\right)=\left(W_{1}, W_{1}\right)$ and $\left(W_{1}^{\prime \prime}, W_{2}^{\prime \prime}\right)=$ $\left(W_{1},-W_{1}\right)$ are three couplings of $(\mu, \mu)$. These three couplings have very different laws. In the one hand, $W_{1}$ and $W_{2}$ are independent and $W_{1} \neq \pm W_{2}$ a.s. and in the other hand $W_{1}^{\prime}=W_{2}^{\prime}$ and $W_{1}^{\prime \prime}=-W_{2}^{\prime \prime}$.

In order to establish (1.2), we remark that it is sufficient to build $\left(X_{1}, X_{2}\right)$ a coupling of $\left(\mathcal{D}\left(X\left(\cdot, x_{1}\right)\right), \mathcal{D}\left(X\left(\cdot, x_{2}\right)\right)\right)$ on $\mathbb{R}^{+}$such that for any $t \geqslant 0$

$$
\mathbb{P}\left(X_{1}(t) \neq X_{2}(t)\right) \leqslant c \mathrm{e}^{-\beta t} .
$$

By induction, it suffices to construct a coupling on a fixed interval [0, $T]$. Indeed, we first set

$$
X_{i}(0)=x_{i}, \quad i=1,2 .
$$

Then we build a probability space $\left(\Omega^{\prime}, \mathcal{F}^{\prime}, \mathbb{P}^{\prime}\right)$ and a measurable function $\left(\omega^{\prime}, t, x_{1}, x_{2}\right) \rightarrow Z_{i}\left(t, x_{1}, x_{2}\right)$ such that for any $\left(x_{1}, x_{2}\right),\left(Z_{i}\left(\cdot, x_{1}, x_{2}\right)\right)_{i=1,2}$ is a coupling of $\left(X\left(\cdot, x_{i}\right)\right)_{i=1,2}$ on $[0, T]$.

The induction argument is then as follows. Assuming that we have built $\left(X_{1}, X_{2}\right)$ on $[0, n T]$, we take $\left(Z_{1}, Z_{2}\right)$ as above independent of $\left(X_{1}, X_{2}\right)$ on $[0, n T]$ and set

$$
X_{i}(n T+t)=Z_{i}\left(t, X_{1}(n T), X_{2}(n T)\right), \quad \text { for } t \in(0, T] .
$$


The Markov property of $X$ implies that $\left(X_{1}, X_{2}\right)$ is a coupling of $\left(\mathcal{D}\left(X\left(\cdot, x_{1}\right)\right), \mathcal{D}\left(X\left(\cdot, x_{2}\right)\right)\right)$ on $[0,(n+1) T]$.

The coupling $\left(Z_{1}, Z_{2}\right)$ on $[0, T]$ constructed below satisfies the following properties

$$
\begin{aligned}
& \mathbb{P}\left(Z_{1}\left(T, x_{1}, x_{2}\right)=Z_{2}\left(T, x_{1}, x_{2}\right)\right) \geqslant p_{0}>0, \quad \text { if } x_{1} \neq x_{2}, \\
& \mathbb{P}\left(Z_{1}\left(\cdot, x_{1}, x_{2}\right)=Z_{2}\left(\cdot, x_{1}, x_{2}\right)\right)=1, \quad \text { if } x_{1}=x_{2} .
\end{aligned}
$$

Invoking (1.5), we obtain that

$$
\mathbb{P}\left(X_{1}(n T) \neq X_{2}(n T) \mid X_{1}((n-1) T)=X_{2}((n-1) T)\right)=0 .
$$

Thus it follows

$$
\begin{aligned}
\mathbb{P}\left(X_{1}(n T) \neq X_{2}(n T)\right) \leqslant & \mathbb{P}\left(X_{1}((n-1) T) \neq X_{2}((n-1) T)\right) \\
& \times \mathbb{P}\left(X_{1}(n T) \neq X_{2}(n T) \mid X_{1}((n-1) T) \neq X_{2}((n-1) T)\right) .
\end{aligned}
$$

We easily get from (1.4) and (1.5)

$$
\mathbb{P}\left(X_{1}(t) \neq X_{2}(t), \text { for some } t \geqslant n T\right) \leqslant\left(1-p_{0}\right)^{n},
$$

which implies (1.3) and allows us to conclude.

Before building $\left(Z_{1}, Z_{2}\right)$ such that (1.4) and (1.5) hold, we need to define some notions. Let $\mu, \mu_{1}$ and $\mu_{2}$ be three probability measures on a space $(E, \mathcal{E})$ such that $\mu_{1}$ and $\mu_{2}$ are absolutely continuous with respect to $\mu$. We set

$$
\begin{aligned}
& d\left|\mu_{1}-\mu_{2}\right|=\left|\frac{\mathrm{d} \mu_{1}}{\mathrm{~d} \mu}-\frac{\mathrm{d} \mu_{2}}{\mathrm{~d} \mu}\right| \mathrm{d} \mu, \\
& d\left(\mu_{1} \wedge \mu_{2}\right)=\left(\frac{\mathrm{d} \mu_{1}}{\mathrm{~d} \mu} \wedge \frac{\mathrm{d} \mu_{2}}{\mathrm{~d} \mu}\right) \mathrm{d} \mu, \\
& d\left(\mu_{1}-\mu_{2}\right)^{+}=\left(\frac{\mathrm{d} \mu_{1}}{\mathrm{~d} \mu}-\frac{\mathrm{d} \mu_{2}}{\mathrm{~d} \mu}\right)^{+} \mathrm{d} \mu .
\end{aligned}
$$

These definitions do not depend on the choice of $\mu$. Moreover we have

$$
\left\|\mu_{1}-\mu_{2}\right\|_{\mathrm{var}}=\frac{1}{2}\left|\mu_{1}-\mu_{2}\right|(E)=\left(\mu_{1}-\mu_{2}\right)^{+}(E)=\frac{1}{2} \int_{E}\left|\frac{\mathrm{d} \mu_{1}}{\mathrm{~d} \mu}-\frac{\mathrm{d} \mu_{2}}{\mathrm{~d} \mu}\right| \mathrm{d} \mu .
$$

The following lemma is the key of our proof.

Lemma 1.2. Let $\left(\mu_{1}, \mu_{2}\right)$ be two probability measures on $(E, \mathcal{E})$. Then

$$
\left\|\mu_{1}-\mu_{2}\right\|_{\text {var }}=\min \mathbb{P}\left(Z_{1} \neq Z_{2}\right) .
$$

The minimum is taken over the coupling $\left(Z_{1}, Z_{2}\right)$ of $\left(\mu_{1}, \mu_{2}\right)$. Such a coupling exists and is called a maximal coupling and has the following property:

$$
\mathbb{P}\left(Z_{1}=Z_{2}, Z_{1} \in \Gamma\right)=\left(\mu_{1} \wedge \mu_{2}\right)(\Gamma) \text { for any } \Gamma \in \mathcal{E} .
$$

The proof of Lemma 1.2 is given in the appendix. We consider $W^{\prime}$ a Wiener process. If $x_{1}=x_{2}=x$, we choose the trivial coupling $\left(Z_{i}(\cdot, x, x)\right)_{i=1,2}$ on $[0, T]$. In other words, we set $Z_{1}(\cdot, x, x)=Z_{2}(\cdot, x, x)=X^{\prime}(\cdot, x)$ on $[0, T]$ where $X^{\prime}(\cdot, x)$ is the solution of (1.1) associated with $W^{\prime}$. Thus (1.5) is clear.

For $x_{1} \neq x_{2}$, the idea is borrowed from [15]. We consider $\left(\widetilde{Z}_{1}\left(\cdot, x_{1}, x_{2}\right), Z_{2}\left(\cdot, x_{1}, x_{2}\right)\right)$ the maximal coupling of $\left(\mathcal{D}\left(X\left(\cdot, x_{1}\right)+\frac{T-\cdot}{T}\left(x_{2}-x_{1}\right)\right), \mathcal{D}\left(X\left(\cdot, x_{2}\right)\right)\right)$ on $[0, T]$ and we set $Z_{1}\left(t, x_{1}, x_{2}\right)=\widetilde{Z}_{1}\left(t, x_{1}, x_{2}\right)-\frac{T-t}{T}\left(x_{2}-x_{1}\right)$. Then it is easy to see that $\left(Z_{i}\left(\cdot, x_{1}, x_{2}\right)\right)_{i=1,2}$ is a coupling of $\left(\mathcal{D}\left(X\left(\cdot, x_{i}\right)\right)_{i=1,2}\right.$ on $[0, T]$ and we have

$$
\mathbb{P}\left(Z_{1}\left(T, x_{1}, x_{2}\right)=Z_{2}\left(T, x_{1}, x_{2}\right)\right) \geqslant \mathbb{P}\left(\widetilde{Z}_{1}\left(\cdot, x_{1}, x_{2}\right)=Z_{2}\left(\cdot, x_{1}, x_{2}\right)\right) .
$$

We need the following result which is Lemma D.1 of [17] 
Lemma 1.3. Let $\mu_{1}$ and $\mu_{2}$ be two probability measures on a space $(E, \mathcal{E})$. Let $A$ be an event of E. Assume that $\mu_{1}^{A}=\mu_{1}(A \cap \cdot)$ is equivalent to $\mu_{2}^{A}=\mu_{2}(A \cap \cdot)$. Then for any $p>1$ and $C>1$

$$
\int_{A}\left(\frac{\mathrm{d} \mu_{1}^{A}}{\mathrm{~d} \mu_{2}^{A}}\right)^{p+1} \mathrm{~d} \mu_{2} \leqslant C<\infty \quad \text { implies }\left(\mu_{1} \wedge \mu_{2}\right)(A) \geqslant\left(1-\frac{1}{p}\right)\left(\frac{\mu_{1}(A)^{p}}{p C}\right)^{1 /(p-1)} .
$$

Using (1.7) and Lemmas 1.2 and 1.3 with $E=C([0, T] ; \Pi)$, we obtain that

$$
\mathbb{P}\left(Z_{1}\left(T, x_{1}, x_{2}\right)=Z_{2}\left(T, x_{1}, x_{2}\right)\right) \geqslant\left(1-\frac{1}{p}\right)\left(p \int_{E}\left(\frac{\mathrm{d} \tilde{\mu}_{1}}{\mathrm{~d} \mu_{2}}\right)^{p+1} \mathrm{~d} \mu_{2}\right)^{-1 /(p-1)},
$$

where $\left(\tilde{\mu}_{1}, \mu_{2}\right)=\left(\mathcal{D}\left(X\left(\cdot, x_{1}\right)+\frac{T-\cdot}{T}\left(x_{2}-x_{1}\right)\right), \mathcal{D}\left(X\left(\cdot, x_{2}\right)\right)\right)$ on $[0, T]$.

We use a Girsanov formula to estimate $\int_{E}\left(\frac{\mathrm{d} \tilde{\mu}_{1}}{\mathrm{~d} \mu_{2}}\right)^{p+1} \mathrm{~d} \mu_{2}$. Setting $\widetilde{X}(t)=X\left(t, x_{1}\right)+\frac{T-t}{T}\left(x_{2}-x_{1}\right)$, we obtain that $\tilde{\mu}_{1}$ is the distribution of $\widetilde{X}$ under the probability $\mathbb{P}$ and that $\widetilde{X}$ is the unique solution of

$$
\frac{\mathrm{d} \tilde{X}}{\mathrm{~d} t}-\frac{1}{T}\left(x_{2}-x_{1}\right)+f\left(\tilde{X}(t)+\frac{T-t}{T}\left(x_{2}-x_{1}\right)\right)=\frac{\mathrm{d} W}{\mathrm{~d} t}, \quad \tilde{X}(0)=x_{2} .
$$

We set $W^{\prime}(t)=W(t)+\int_{0}^{t} R d(s) \mathrm{d} t$, where

$$
d(t)=\frac{1}{T}\left(x_{2}-x_{1}\right)+f(\tilde{X}(t))-f\left(\tilde{X}(t)+\frac{T-t}{T}\left(x_{2}-x_{1}\right)\right) .
$$

Then $\tilde{X}$ is a solution of

$$
\frac{\mathrm{d} \tilde{X}}{\mathrm{~d} t}+f(\tilde{X})=\frac{\mathrm{d} W^{\prime}}{\mathrm{d} t}, \quad \tilde{X}(0)=x_{2}
$$

We are working on the torus and $f$ is continuous, therefore $d$ is uniformly bounded:

$$
|d(t)| \leqslant \frac{1}{T}+2|f|_{\infty}
$$

Hence, the Novikov condition is satisfied and the Girsanov formula can be applied. Then we set

$$
\mathrm{dP}^{\prime}=\exp \left(\int_{0}^{t} d(s) \mathrm{d} W(s)-\frac{1}{2} \int_{0}^{t}|d(s)|^{2} \mathrm{~d} t\right) \mathrm{d} \mathbb{P} .
$$

We deduce from the Girsanov formula that $\mathbb{P}^{\prime}$ is a probability measure under which $W^{\prime}$ is a Brownian motion and $\widetilde{X}$ is a solution of (1.10), then the law of $\widetilde{X}$ under $\mathbb{P}^{\prime}$ is $\mu_{2}$. Moreover

$$
\int_{E}\left(\frac{\mathrm{d} \tilde{\mu}_{1}}{\mathrm{~d} \mu_{2}}\right)^{p+1} \mathrm{~d} \mu_{2} \leqslant \exp \left(c_{p}\left(\frac{1}{T}+|f|_{\infty}^{2} T\right)\right)
$$

which allows us to conclude this example. Indeed, by applying (1.7), (1.8) and (1.11) we get (1.4).

\subsection{A representative two-dimensional example}

The example we consider now is a two-dimensional system which mimics the decomposition of a stochastic partial differential equation according to low and high modes of the solution. This example allows the introduction of the main ideas in a simplified context, the system has the form

$$
\left\{\begin{array}{l}
\mathrm{d} X+2 X \mathrm{~d} t+f(X, Y) \mathrm{d} t=\sigma_{l}(X) \mathrm{d} \beta, \\
\mathrm{d} Y+2 Y \mathrm{~d} t+g(X, Y) \mathrm{d} t=\sigma_{h}(X) \mathrm{d} \eta, \\
X(0)=x_{0}, \quad Y(0)=y_{0} .
\end{array}\right.
$$


We set $u=(X, Y)$ and $W=(\beta, \eta)$. We use the following assumptions

$$
\left\{\begin{array}{l}
\text { (i) } f, g, \sigma_{l} \text { and } \sigma_{h} \text { are bounded and Lipschitz. } \\
\text { (ii) There exists } K_{0}>0 \text { such that, } \\
f(x, y) x+g(x, y) y \geqslant-\left(|x|^{2}+|y|^{2}+K_{0}\right), \quad(x, y) \in \mathbb{R}^{2} .
\end{array}\right.
$$

Condition (i) ensures existence and uniqueness of a solution to (1.12) once the initial data $u_{0}=\left(x_{0}, y_{0}\right)$ is given. It is also classical that weak existence and uniqueness holds. We denote by $X\left(\cdot, u_{0}\right), Y\left(\cdot, u_{0}\right), u\left(\cdot, u_{0}\right)$ the solution where $u_{0}=\left(x_{0}, y_{0}\right)$ and $u=(X, Y)$. Moreover, it is easy to see that, by (ii), there exists an invariant measure $v$.

Contrary to Section 1.1, we want to allow degenerate noises. More precisely, we want to treat the case when the noise on the second equation may vanish. This possible degeneracy is compensated by a dissipativity assumption. We use the following assumptions.

$$
\left\{\begin{array}{l}
\text { (i) There exists } \sigma_{0}>0 \text { such that, } \sigma_{l}(x) \geqslant \sigma_{0}, x \in \mathbb{R} . \\
\text { (ii) }\left|g\left(x, y_{1}\right)-g\left(x, y_{2}\right)\right| \leqslant\left|y_{1}-y_{2}\right|,\left(x, y_{1}, y_{2}\right) \in \mathbb{R}^{2} .
\end{array}\right.
$$

By the dissipativity method (see [4], Section 11.5), (ii) implies exponential convergence to equilibrium for the second equation if $X$ is fixed. Whilst the coupling argument explained in Section 1.1 can be used to treat the first equation when $Y$ is fixed. Note however that we need a more sophisticated coupling here. Indeed, the simple coupling explained above seems to be useful only for additive noise.

Here, we explain how these two arguments may be coupled to treat system (1.12). The essential tool which allows to treat system (1.12) is the so-called Foias-Prodi estimate which reflects the dissipativity property of the second equation. It is a simple consequence of (1.14)(ii).

Proposition 1.4. Let $\left(u_{i}, W_{i}\right)_{i=1,2}$ be two weak solutions of (1.12) such that

$$
X_{1}(s)=X_{2}(s), \quad \eta_{1}(s)=\eta_{2}(s), \quad s \in[0, t],
$$

then

$$
\left|u_{1}(t)-u_{2}(t)\right| \leqslant\left|u_{1}(0)-u_{2}(0)\right| \mathrm{e}^{-t}
$$

Since the noise on the second equation might be degenerate, there is no hope to use Girsanov formula on the full system. We can use it to modify the drift of the first equation only and it is not possible to derive a strong estimate as (1.3).

Recall that in Section 1.1, we have built the coupling $\left(X_{1}, X_{2}\right)$ of $\left(\mathcal{D}\left(X\left(\cdot, x_{0}^{1}\right)\right), \mathcal{D}\left(X\left(\cdot, x_{0}^{2}\right)\right)\right)$ by induction on $[0, k T]$ by using a coupling $\left(Z_{i}\left(\cdot, x_{0}^{1}, x_{0}^{2}\right)\right)_{i=1,2}$ of $\left(\mathcal{D}\left(X\left(\cdot, x_{0}^{i}\right)\right)\right)_{i=1,2}$ on $[0, T]$ which satisfies $(1.5)$. Then if $\left(X_{1}, X_{2}\right)$ were coupled at time $k T,\left(X_{1}, X_{2}\right)$ would be coupled on $[k T, \infty)$ with probability one. Thus to conclude, it was sufficient to establish (1.4).

In this section, since we couple $\left(X_{1}, X_{2}\right)$, but not $\left(Y_{1}, Y_{2}\right)$, then there is no hope that a couple $\left(X_{1}, X_{2}\right)$ coupled at time $k T$ remains coupled at time $(k+1) T$ with probability one.

However, coupling the $X$ 's and using Foias-Prodi estimates, we obtain a coupling $\left(u_{1}, u_{2}\right)$ of $\left(\mathcal{D}\left(u\left(\cdot, u_{0}^{1}\right)\right)\right.$, $\left.\mathcal{D}\left(u\left(\cdot, u_{0}^{2}\right)\right)\right)$ on $\mathbb{R}^{+}$such that

$$
\mathbb{P}\left(\left|u_{1}(t)-u_{2}(t)\right|>c \mathrm{e}^{-\beta t}\right) \leqslant c \mathrm{e}^{-\beta t}\left(1+\left|u_{0}^{1}\right|^{2}+\left|u_{0}^{2}\right|^{2}\right) .
$$

This estimate does not imply the decay of the total variation of $\mathcal{P}_{t}^{*} \delta_{u_{0}^{1}}-\mathcal{P}_{t}^{*} \delta_{u_{0}^{2}}$, but the decay of this quantity in the Wasserstein distance $|\cdot|_{\mathrm{Lip}_{b}}^{*}$ which is the dual norm of the Lipschitz and bounded functions. Indeed, for $\psi$ Lipschitz and bounded, we clearly have

$$
\begin{aligned}
\left|\mathbb{E} \psi\left(u\left(t, u_{0}^{1}\right)\right)-\mathbb{E} \psi\left(u\left(t, u_{0}^{2}\right)\right)\right| & =\left|\mathbb{E} \psi\left(u_{1}(t)\right)-\mathbb{E} \psi\left(u_{2}(t)\right)\right|, \\
& \leqslant 2|\psi|_{\infty} \mathbb{P}\left(\left|u_{1}(t)-u_{2}(t)\right|>c \mathrm{e}^{-\beta t}\right)+|\psi|_{\text {Lip }} c \mathrm{e}^{-\beta t},
\end{aligned}
$$

and then by (1.15)

$$
\left|\mathbb{E} \psi\left(u\left(t, u_{0}^{1}\right)\right)-\mathbb{E} \psi\left(u\left(t, u_{0}^{1}\right)\right)\right| \leqslant c|\psi|_{\operatorname{Lip}_{b}} \mathrm{e}^{-\beta t}\left(1+\left|u_{0}^{1}\right|^{2}+\left|u_{0}^{2}\right|^{2}\right) .
$$


The idea of the proof is the following. We couple $\left(\mathcal{D}\left(X\left(\cdot, u_{0}^{i}\right), \eta\right)\right)_{i=1,2}$. Then using the Foias-Prodi estimate, we control $Y_{1}-Y_{2}$ which is equivalent to control $u_{1}-u_{2}$. By controlling $u_{1}-u_{2}$, we control the probability to remain coupled.

Remark 1.5. In the general case $f, g$ are not globally Lipschitz and bounded and a cut-off has to be used. This further difficulty will be treated in the context of the CGL equation below.

It is convenient to introduce the following functions:

$$
l_{0}(k)=\min \left\{l \in\{0, \ldots, k\} \mid P_{l, k}\right\},
$$

where $\min \phi=\infty$ and

$$
\left(P_{l, k}\right) \quad\left\{\begin{array}{l}
X_{1}(t)=X_{2}(t), \quad \eta_{1}(t)=\eta_{2}(t), \quad \forall t \in[l T, k T] \\
\left|u_{i}(l T)\right| \leqslant d^{*}, \quad i=1,2
\end{array}\right.
$$

The first requirement in $\left(P_{l, k}\right)$ states that the two solutions of the first equation are coupled on $[l T, k T]$. Notice that Proposition 1.4 gives

$$
l_{0}(k)=l \quad \text { implies } \quad\left|u_{1}(t)-u_{2}(t)\right| \leqslant 2 d^{*} \mathrm{e}^{-(t-l T)}, \quad \text { for any } t \in[l T, k T] .
$$

From now on we say that $\left(X_{1}, X_{2}\right)$ are coupled at $k T$ if $l_{0}(k) \leqslant k$, in other words if $l_{0}(k) \neq \infty$.

We set

$$
d_{0}=4\left(d^{*}\right)^{2} \text {. }
$$

We prove the two following properties.

For any $d_{0}>0$

$$
\left\{\begin{array}{l}
\exists p_{0}\left(d_{0}\right)>0,\left(p_{i}\right)_{i} \geqslant 1, T_{0}\left(d_{0}\right)>0 \text { such that for any } l \leqslant k, \\
\mathbb{P}\left(l_{0}(k+1)=l \mid l_{0}(k)=l\right) \geqslant p_{k-l}, \text { for any } T \geqslant T_{0}\left(d_{0}\right), \\
1-p_{i} \leqslant \mathrm{e}^{-i T}, \quad i \geqslant 1,
\end{array}\right.
$$

and, for any $\left(R_{0}, d_{0}\right)$ sufficiently large,

$$
\left\{\begin{array}{l}
\exists T^{*}\left(R_{0}\right)>0 \text { and } p_{-1}>0 \text { such that for any } T \geqslant T^{*}\left(R_{0}\right) \\
\mathbb{P}\left(l_{0}(k+1)=k+1 \mid l_{0}(k)=\infty, \mathcal{H}_{k} \leqslant R_{0}\right) \geqslant p_{-1}
\end{array}\right.
$$

where

$$
\mathcal{H}_{k}=\left|u_{1}(k T)\right|^{2}+\left|u_{2}(k T)\right|^{2} .
$$

(1.18) states that the probability that two solutions decouples at $k T$ is very small, (1.19) states that, inside a ball, the probability that two solutions get coupled at $(k+1) T$ is uniformly bounded below.

In the particular case where $\sigma_{l}(x)$ does not depend on $x$ and where $K_{0}=0$, one can apply a similar proof as in Section 1.1 to establish a result closely related to (1.18), (1.19). This technic has been developed in [15]. But it does not seem to work in the general case.

Consequently, we use some tools developed in [17] to establish (1.18), (1.19). Note that in (1.19), we use only starting points in a ball of radius $R_{0}$. This is due to the fact that to prove (1.19), we need to estimate some terms which cannot be controlled on $\mathbb{R}^{2}$ but only inside a ball. This further difficulty is due to the fact that contrary to the simple example of Section 1.1, we work on an unbounded phase space and is overcomed thanks to another ingredient which is the so-called Lyapounov structure. It allows the control of the probability to enter the ball of radius $R_{0}$. In our example, it is an easy consequence of (1.13)(ii). More precisely, we use the property that for any solution $u\left(\cdot, u_{0}\right)$

$$
\left\{\begin{array}{l}
\mathbb{E}\left|u\left(t, u_{0}\right)\right|^{2} \leqslant \mathrm{e}^{-2 t}\left|u_{0}\right|^{2}+\frac{K_{1}}{2}, \\
\mathbb{E}\left(\left|u\left(\tau^{\prime}, u_{0}\right)\right|^{4} 1_{\tau^{\prime}<\infty}\right) \leqslant K^{\prime}\left(\left|u_{0}\right|^{4}+1\right),
\end{array}\right.
$$

for any stopping times $\tau^{\prime}$.

The following proposition is a consequence of Theorem 1.8 given in a more general setting below. 
Proposition 1.6. If there exists a coupling of $\mathcal{D}\left(u\left(\cdot, u_{0}^{i}\right), W\right)$ such that (1.18), (1.19) are satisfied, then (1.15) is true. Thus there exists a unique invariant measure $v$ of $\left(\mathcal{P}_{t}\right)_{t}$. Moreover there exist $C$ and $\alpha$ such that

$$
\left\|\mathcal{P}_{t} \mu-v\right\|_{\operatorname{Lip}_{b}\left(\mathbb{R}^{2}\right)}^{*} \leqslant C \mathrm{e}^{-\alpha t}\left(1+\int_{\mathbb{R}^{2}}|u| \mathrm{d} \mu(u)\right) .
$$

To obtain (1.18) and (1.19), we introduce three more ingredients. First in order to build a coupling $\left(\left(u_{1}, W_{1}\right)\right.$, $\left.\left(u_{2}, W_{2}\right)\right)$ such that $\left(\left(X_{1}, \eta_{1}\right),\left(X_{2}, \eta_{2}\right)\right)$ is a maximal coupling, we use the following results contained in [17], although not explicitly stated. Its proof is postponed to the appendix.

Proposition 1.7. Let $E$ and $F$ be two polish spaces, $f_{0}: E \rightarrow F$ be a measurable map and $\left(\mu_{1}, \mu_{2}\right)$ be two probability measures on E. We set

$$
v_{i}=f_{0}^{*} \mu_{i}, \quad i=1,2 .
$$

Then there exist a coupling $\left(V_{1}, V_{2}\right)$ of $\left(\mu_{1}, \mu_{2}\right)$ such that $\left(f_{0}\left(V_{1}\right), f_{0}\left(V_{2}\right)\right)$ is a maximal coupling of $\left(v_{1}, v_{2}\right)$.

We also remark that given $(X, \eta)$ on $[0, T]$, there exists a unique solution $Y\left(\cdot, u_{0}\right)$ of

$$
\mathrm{d} Y+2 Y \mathrm{~d} t+g(X, Y) \mathrm{d} t=\sigma_{h}(X) \mathrm{d} \eta, \quad Y\left(0, u_{0}\right)=y_{0} .
$$

We set

$$
Y\left(\cdot, u_{0}\right)=\Phi\left(X, \eta, u_{0}\right)(\cdot) .
$$

It is easy to see that $Y$ is adapted to the filtration associated to $\eta$ and $X$.

Proposition 1.4 implies that for any given $(X, \eta)$

$$
\left|\Phi\left(X, \eta, u_{0}^{1}\right)(t)-\Phi\left(X, \eta, u_{0}^{2}\right)(t)\right| \leqslant \mathrm{e}^{-t}\left|u_{0}^{1}-u_{0}^{2}\right|
$$

Then we rewrite the equation for $X$ as follows

$$
\left\{\begin{array}{l}
\mathrm{d} X+2 X \mathrm{~d} t+f\left(X, \Phi\left(X, \eta, u_{0}\right)\right) \mathrm{d} t=\sigma_{l}(X) \mathrm{d} \beta \\
X(0)=x_{0}
\end{array}\right.
$$

The Girsanov formula can then be used on (1.22) as in Section 1.1.

We finally remark that by induction, it suffices to construct a probability space $\left(\Omega_{0}, \mathcal{F}_{0}, \mathbb{P}_{0}\right)$ and two measurable couples of functions $\left(\omega_{0}, u_{0}^{1}, u_{0}^{2}\right) \rightarrow\left(V_{i}\left(\cdot, u_{0}^{1}, u_{0}^{2}\right)\right)_{i=1,2}$ and $\left(V_{i}^{\prime}\left(\cdot, u_{0}^{1}, u_{0}^{2}\right)\right)_{i=1,2}$ and such that, for any $\left(u_{0}^{1}, u_{0}^{2}\right)$, $\left(V_{i}\left(\cdot, u_{0}^{1}, u_{0}^{2}\right)\right)_{i=1,2}$ and $\left(V_{i}^{\prime}\left(\cdot, u_{0}^{1}, u_{0}^{2}\right)\right)_{i=1,2}$ are two couplings of $\left(\mathcal{D}\left(u\left(\cdot, u_{0}^{i}\right), W\right)\right)_{i=1,2}$ on $[0, T]$. Indeed, we first set

$$
u_{i}(0)=u_{0}^{i}, \quad W_{i}(0)=0, \quad i=1,2 .
$$

Assuming that we have built $\left(u_{i}, W_{i}\right)_{i=1,2}$ on $[0, k T]$, then we take $\left(V_{i}\right)_{i}$ and $\left(V_{i}^{\prime}\right)_{i}$ as above independent of $\left(u_{i}, W_{i}\right)_{i=1,2}$ on $[0, k T]$ and set

$$
\left(u_{i}(k T+t), W_{i}(k T+t)\right)= \begin{cases}V_{i}\left(t, u_{1}(k T), u_{2}(k T)\right) & \text { if } l_{0}(k) \leqslant k, \\ V_{i}^{\prime}\left(t, u_{1}(k T), u_{2}(k T)\right) & \text { if } l_{0}(k)=\infty,\end{cases}
$$

for any $t \in[0, T]$.

Proof of (1.18). To build $\left(V_{i}\left(\cdot, u_{0}^{1}, u_{0}^{2}\right)\right)_{i=1,2}$, we apply Proposition 1.7 to $E=C\left((0, T) ; \mathbb{R}^{2}\right)^{2}, F=C((0, T) ; \mathbb{R})^{2}$,

$$
f_{0}(u, W)=(X, \eta), \quad \text { where } u=\left(\begin{array}{c}
X \\
Y
\end{array}\right), W=\left(\begin{array}{l}
\beta \\
\eta
\end{array}\right),
$$

and to

$$
\mu_{i}=\mathcal{D}\left(u\left(\cdot, u_{0}^{i}\right), W\right), \quad \text { on }[0, T] .
$$

Remark that if we set $v_{i}=f_{0}^{*} \mu_{i}$, we obtain

$$
v_{i}=\mathcal{D}\left(X\left(\cdot, u_{0}^{i}\right), \eta\right), \quad \text { on }[0, T] .
$$


We write

$$
\left(Z_{i}, \xi_{i}\right)=f_{0}\left(V_{i}\right), \quad i=1,2 .
$$

Then $\left(V_{i}\left(\cdot, u_{0}^{1}, u_{0}^{2}\right)\right)_{i=1,2}$ is a coupling of $\left(\mu_{1}, \mu_{2}\right)$ such that $\left(\left(Z_{i}, \xi_{i}\right)\left(\cdot, u_{0}^{1}, u_{0}^{2}\right)\right)_{i=1,2}$ is a maximal coupling of $\left(v_{1}, v_{2}\right)$.

We first use a Girsanov formula to estimate $I_{p}$, where

$$
I_{p}=\int_{F}\left(\frac{\mathrm{d} \nu_{2}}{\mathrm{~d} \nu_{1}}\right)^{p+1} \mathrm{~d} \nu_{2} .
$$

Then, using Lemma 1.2, we establish (1.18).

We consider a couple $\left(u_{i}, W_{i}\right)_{i=1,2}$ consisting of two solutions of (1.12) on [0,kT]. From now on, we are only concerned with a trajectory of $\left(u_{i}, W_{i}\right)_{i=1,2}$ such that $l_{0}(k)=l \leqslant k$. We set

$$
x=X_{1}(k T)=X_{2}(k T), \quad y_{i}=Y_{i}(k T), \quad i=1,2 .
$$

Let $(\beta, \xi)$ be a two-dimensional Brownian motion defined on a probability space $(\Omega, \mathcal{F}, \mathbb{P})$. We denote by $Z$ the unique solution of

$$
\left\{\begin{array}{l}
\mathrm{d} Z+2 Z \mathrm{~d} t+f\left(Z, \Phi\left(Z(\cdot), \xi(\cdot),\left(x, y_{1}\right)\right)\right) \mathrm{d} t=\sigma_{l}(Z) \mathrm{d} \beta \\
Z(0)=x
\end{array}\right.
$$

Taking into account (1.24), we obtain that $v_{1}$ is the distribution of $(Z, \xi)$ under the probability $\mathbb{P}$.

We set $\tilde{\beta}(t)=\beta(t)+\int_{0}^{t} d(s) \mathrm{d} t$ where

$$
d(t)=\frac{1}{\sigma_{l}(Z(t))}\left(f\left(Z(t), \Phi\left(Z, \xi,\left(x, y_{2}\right)\right)(t)\right)-f\left(Z(t), \Phi\left(Z, \xi,\left(x, y_{1}\right)\right)(t)\right)\right) .
$$

Then $Z$ is a solution of

$$
\left\{\begin{array}{l}
\mathrm{d} Z+2 Z \mathrm{~d} t+f\left(Z, \Phi\left(Z(\cdot), \xi(\cdot),\left(x, y_{2}\right)\right)\right) \mathrm{d} t=\sigma_{l}(Z) \mathrm{d} \tilde{\beta} \\
Z(0)=x
\end{array}\right.
$$

Since $f$ is bounded and $\sigma_{l}$ is bounded below, then $d$ is uniformly bounded. Hence, the Novikov condition is satisfied and the Girsanov formula can be applied. Then we set

$$
\mathrm{d} \widetilde{\mathbb{P}}=\exp \left(\int_{0}^{T} d(s) \mathrm{d} W(s)-\frac{1}{2} \int_{0}^{T}|d(s)|^{2} \mathrm{~d} t\right) \mathrm{d} \mathbb{P} .
$$

We deduce from the Girsanov formula that $\widetilde{\mathbb{P}}$ is a probability under which $(\tilde{\beta}, \xi)$ is a Brownian motion and since $Z$ is a solution of (1.26), then the law of $(Z, \xi)$ under $\mathbb{P}$ is $v_{2}$. Moreover

$$
I_{p} \leqslant \mathbb{E} \exp \left(c_{p} \int_{0}^{T}|d(s)|^{2} \mathrm{~d} t\right) .
$$

Since $f$ is Lipschitz, then we infer from (1.25) and (1.14)i) that

$$
|d(t)| \leqslant \sigma_{0}^{-1}|f|_{\text {Lip }}\left|\Phi\left(Z(\cdot), \xi(\cdot),\left(x, y_{1}\right)\right)(t)-\Phi\left(Z(\cdot), \xi(\cdot),\left(x, y_{2}\right)\right)(t)\right| .
$$

Now we use the Foias-Prodi estimate. Applying (1.17) and (1.21), it follows from $l_{0}(k)=l$ that

$$
|d(t)|^{2} \leqslant d_{0} \sigma_{0}^{-2}|f|_{\text {Lip }}^{2} \exp (-2(k-l) T)
$$

Then it follows that

$$
I_{p} \leqslant \exp \left(c_{p} \sigma_{0}^{-2} d_{0}|f|_{\text {Lip }}^{2} \mathrm{e}^{-2(k-l) T}\right) .
$$


Note that

$$
\left\|v_{1}-v_{2}\right\|_{\mathrm{var}}=\int_{F}\left|\frac{\mathrm{d} \nu_{2}}{\mathrm{~d} \nu_{1}}-1\right| \mathrm{d} \nu_{2} \leqslant \sqrt{\int\left(\frac{\mathrm{d} \nu_{2}}{\mathrm{~d} \nu_{1}}\right)^{2} \mathrm{~d} \nu_{2}-1} .
$$

We infer from (1.28) that, for $T \geqslant T_{0}\left(d_{0}\right)=\left(\sigma_{0}^{-2} c_{p} d_{0}|f|_{\text {Lip }}^{2}\right)^{-1}$,

$$
\left\|v_{1}-v_{2}\right\|_{\mathrm{var}} \leqslant \mathrm{e}^{-(k-l) T} .
$$

Applying Lemma 1.2 to the maximal coupling $\left(Z_{1}, Z_{2}\right)_{i=1,2}$ of $\left(v_{1}, v_{2}\right)$ gives

$$
\mathbb{P}\left(\left(Z_{1}, \xi_{1}\right) \neq\left(Z_{2}, \xi_{2}\right)\right) \leqslant\left\|v_{1}-v_{2}\right\|_{\text {var }} \leqslant \mathrm{e}^{-(k-l) T} .
$$

Using (1.23) and (1.29), we obtain that on $l_{0}(k)=l$

$$
\mathbb{P}\left(\left(X_{1}, \eta_{1}\right) \neq\left(X_{2}, \eta_{2}\right) \text { on }[k T,(k+1) T] \mid \mathcal{F}_{k T}\right) \leqslant \mathrm{e}^{-(k-l) T} .
$$

Noticing that

$$
\left\{l_{0}(k+1)=l\right\}=\left\{l_{0}(k)=l\right\} \cap\left\{\left(X_{1}, \eta_{1}\right)=\left(X_{2}, \eta_{2}\right) \text { on }[k T,(k+1) T]\right\}
$$

and integrating over $l_{0}(k)=l$ gives for $T \geqslant T_{0}(d)$ and for $k>l$

$$
\mathbb{P}\left(l_{0}(k+1) \neq l \mid l_{0}(k)=l\right) \leqslant \mathrm{e}^{-(k-l) T} .
$$

Now, it remains to consider the case $k=l$, we apply Lemmas 1.2 and 1.3 to $\left(Z_{i}, \xi_{i}\right)_{i=1,2}$ which gives

$$
\mathbb{P}\left(\left(Z_{1}, \xi_{1}\right)=\left(Z_{2}, \xi_{2}\right)\right)=\left(v_{1} \wedge v_{2}\right)(F) \geqslant\left(1-\frac{1}{p}\right)\left(p I_{p}\right)^{-1 /(p-1)}
$$

Applying (1.27) and fixing $p>1$, we obtain

$$
\mathbb{P}\left(\left(Z_{1}, \xi_{1}\right)=\left(Z_{2}, \xi_{2}\right)\right) \geqslant p_{0}\left(d_{0}\right)=\left(1-\frac{1}{p}\right) p^{-1 /(p-1)} \exp \left(-c_{p} d_{0}|f|_{\text {Lip }}^{2}\right) .
$$

To conclude, we notice that (1.30) and (1.31) imply (1.18).

Proof of (1.19). Assume that we have $d_{0}>0, \tilde{p}>0, T_{1}>0, R_{1}>4 K_{1}$ and a coupling $\left(\widetilde{V}_{i}\left(\cdot, u_{0}^{1}, u_{0}^{2}\right)\right)_{i=1,2}$ of $\left(\mu_{1}, \mu_{2}\right)$, where

$$
\mu_{i}=\mathcal{D}\left(u\left(\cdot, u_{0}^{i}\right), W\right), \quad \text { on }\left[0, T_{1}\right], i=1,2,
$$

and such that for any $\left(u_{0}^{1}, u_{0}^{2}\right)$ which satisfies $\left|u_{0}^{1}\right|^{2}+\left|u_{0}^{2}\right|^{2} \leqslant R_{1}$

$$
\mathbb{P}\left(Z_{1}\left(T_{1}, u_{0}^{1}, u_{0}^{2}\right)=Z_{2}\left(T_{1}, u_{0}^{1}, u_{0}^{2}\right), \sum_{i=1}^{2}\left|u_{i}\left(T_{1}, u_{0}^{1}, u_{0}^{2}\right)\right|^{2} \leqslant d_{0}\right) \geqslant \tilde{p},
$$

where

$$
\widetilde{V}_{i}\left(\cdot, u_{0}^{1}, u_{0}^{2}\right)=\left(u_{i}\left(\cdot, u_{0}^{1}, u_{0}^{2}\right), W_{i}\left(\cdot, u_{0}^{1}, u_{0}^{2}\right)\right), u_{i}\left(\cdot, u_{0}^{1}, u_{0}^{2}\right)=\left(\begin{array}{c}
Z_{i} \\
G_{i}
\end{array}\right), \quad i=1,2 .
$$

By applying the Lyapounov structure (1.20), we obtain that for any $\theta \geqslant T_{2}\left(R_{0}, R_{1}\right)$

$$
\mathbb{P}\left(\left|u\left(\theta, u_{0}\right)\right|^{2} \geqslant \frac{R_{1}}{2}\right) \leqslant \frac{1}{4}, \quad \text { for any } u_{0} \text { such that }\left|u_{0}\right|^{2} \leqslant \frac{R_{0}}{2} .
$$

In order to build $\left(V_{1}^{\prime}, V_{2}^{\prime}\right)$ such that (1.19) happens, we set $T^{*}\left(R_{0}\right)=T_{1}+T_{2}\left(R_{0}\right)$ and for any $T \geqslant T^{*}\left(R_{0}\right)$, we set $\theta=T-T_{1}$ and we remark that $\theta \geqslant T_{2}\left(R_{0}\right)$. Then we construct the trivial coupling $\left(V_{1}^{\prime \prime}, V_{2}^{\prime \prime}\right)$ on $[0, \theta]$. Finally, we consider $\left(\widetilde{V}_{1}, \widetilde{V}_{2}\right)$ as above independent of $\left(V_{1}^{\prime \prime}, V_{2}^{\prime \prime}\right)$ and we set

$$
V_{i}^{\prime}\left(t, u_{0}^{1}, u_{0}^{2}\right)= \begin{cases}V_{i}^{\prime \prime}\left(t, u_{0}^{1}, u_{0}^{2}\right) & \text { if } t \leqslant \theta, \\ \widetilde{V}_{i}\left(t-\theta, V_{1}^{\prime \prime}\left(\theta, u_{0}^{1}, u_{0}^{2}\right), V_{2}^{\prime \prime}\left(\theta, u_{0}^{1}, u_{0}^{2}\right)\right) & \text { if } t \geqslant \theta .\end{cases}
$$


Combining (1.32) and (1.33), we obtain (1.19) with $p_{-1}=\frac{1}{2} \tilde{p}$.

To build $\left(\widetilde{V}_{i}\left(\cdot, u_{0}^{1}, u_{0}^{2}\right)\right)_{i=1,2}$, we apply Proposition 1.7 to $E=C\left(\left(0, T_{1}\right) ; \mathbb{R}^{2}\right)^{2}, F=\mathbb{R}$,

$$
f_{0}(u, W)=X\left(T_{1}\right), \quad \text { where } u=\left(\begin{array}{c}
X \\
Y
\end{array}\right), W=\left(\begin{array}{c}
\beta \\
\eta
\end{array}\right),
$$

and to $\left(\mu_{1}, \mu_{2}\right)$. Remark that if we set $v_{i}=f_{0}^{*} \mu_{i}$, we obtain

$$
v_{i}=\mathcal{D}\left(X\left(T_{1}, u_{0}^{i}\right)\right) .
$$

Then $\left(\widetilde{V}_{i}\left(\cdot, u_{0}^{1}, u_{0}^{2}\right)\right)_{i=1,2}$ is a coupling of $\left(\mu_{1}, \mu_{2}\right)$ such that $\left(Z_{i}\left(T_{1}, u_{0}^{1}, u_{0}^{2}\right)\right)_{i=1,2}$ is a maximal coupling of $\left(v_{1}, v_{2}\right)$.

Now we notice that if we have $\left(\hat{v}_{1}, \hat{v}_{2}\right)$ two equivalent measures such that $v_{i}$ is equivalent to $\hat{v}_{i}$ for $i=1,2$, then by applying two Schwartz inequality, we obtain that

$$
I_{p} \leqslant\left(J_{2 p+2}^{1}\right)^{1 / 2}\left(J_{4 p}^{2}\right)^{1 / 4}\left(\hat{I}_{4 p+2}\right)^{1 / 4}
$$

where $A=\left[-d_{1}, d_{1}\right]$ and

$$
\begin{array}{rlrl}
I_{p} & =\int_{A}\left(\frac{\mathrm{d} \nu_{1}}{\mathrm{~d} \nu_{2}}\right)^{p+1} \mathrm{~d} \nu_{2}, & J_{p}^{1}=\int_{A}\left(\frac{\mathrm{d} \nu_{1}}{\mathrm{~d} \hat{v}_{1}}\right)^{p} \mathrm{~d} \hat{v}_{1}, \\
\hat{I}_{p}=\int_{A}\left(\frac{\mathrm{d} \hat{v}_{1}}{\mathrm{~d} \hat{v}_{2}}\right)^{p} \mathrm{~d} \hat{v}_{2}, & J_{p}^{2}=\int_{A}\left(\frac{\mathrm{d} \hat{v}_{2}}{\mathrm{~d} \nu_{2}}\right)^{p} \mathrm{~d} \hat{v}_{2} .
\end{array}
$$

Recall that $Z_{i}$ the unique solution of

$$
\left\{\begin{array}{l}
\mathrm{d} Z_{i}+2 Z_{i} \mathrm{~d} t+f\left(Z_{i}, \Phi\left(Z_{i}(\cdot), \xi_{i}(\cdot), u_{0}^{i}\right)\right) \mathrm{d} t=\sigma_{l}\left(Z_{i}\right) \mathrm{d} \beta_{i}, \\
Z_{i}(0)=x_{0}^{i} .
\end{array}\right.
$$

We set $\tilde{\beta}_{i}(t)=\beta_{i}(t)+\int_{0}^{t} d_{i}(s) \mathrm{d} t$ where

$$
d_{i}(t)=-\frac{1}{\sigma_{l}\left(Z_{i}(t)\right)} f\left(Z_{i}(t), \Phi\left(Z_{i}(\cdot), \xi_{i}(\cdot), u_{0}^{i}\right)(t)\right) .
$$

Then $Z_{i}$ is a solution of

$$
\left\{\begin{array}{l}
\mathrm{d} Z_{i}+2 Z_{i} \mathrm{~d} t=\sigma_{l}\left(Z_{i}\right) \mathrm{d} \tilde{\beta}_{i} \\
Z_{i}(0)=x_{0}^{i}
\end{array}\right.
$$

Since $f$ is bounded and $\sigma_{l}$ is bounded below, then $d_{i}$ is uniformly bounded. Hence, the Novikov condition is satisfied and the Girsanov formula can be applied. Then we set

$$
\mathrm{d}_{i}=\exp \left(\int_{0}^{T} d_{i}(s) \mathrm{d} W(s)-\frac{1}{2} \int_{0}^{T}\left|d_{i}(s)\right|^{2} \mathrm{~d} t\right) \mathrm{d} \mathbb{P} .
$$

We deduce from the Girsanov formula that $\widetilde{\mathbb{P}}_{i}$ is a probability under which $\left(\tilde{\beta}_{i}, \xi_{i}\right)$ is a Brownian motion. We denote by $\hat{v}_{i}$ the law of $Z_{i}\left(T_{1}\right)$ under $\widetilde{\mathbb{P}}_{i}$. Moreover

$$
J_{p}^{i} \leqslant \exp \left(c_{p} \int_{0}^{T}\left|d_{i}(s)\right|^{2} \mathrm{~d} t\right) \leqslant \exp \left(c_{p} \sigma_{0}^{-2}|f|_{\infty}^{2}\right) .
$$

It is classical that since $\sigma_{l}$ is bounded below, then $\hat{v}_{i}$ has a density $q\left(x_{0}^{i}, z\right)$ with respect to Lebesgue measure $\mathrm{d} z$, that $q$ is continuous with respect to the couple $\left(x_{0}^{i}, z\right)$, where $x_{0}^{i}$ is the initial value and where $z$ is the target value and that $q>0$. Then, we can bound $q$ and $q^{-1}$ uniformly on $\left|x_{0}^{i}\right| \leqslant R_{1}$ and $z \in A=\left[-d_{1}, d_{1}\right]$, which allows us to bound $\hat{I}_{p}$ and then $I_{p}$. Actually:

$$
I_{p} \leqslant C^{\prime}\left(p, d_{1}, T_{1}, R_{1}\right)<\infty .
$$


Now we apply Lemmas 1.3 and 1.2:

$$
\mathbb{P}\left(Z_{1}\left(T_{1}\right)=Z_{2}\left(T_{1}\right),\left|Z_{1}\left(T_{1}\right)\right| \leqslant d_{1}\right) \geqslant\left(1-\frac{1}{p}\right) p^{-1 /(p-1)} I_{p}^{-1 /(p-1)} v_{1}\left(\left[-d_{1}, d_{1}\right]\right)^{p /(p-1)} .
$$

If we fix $d_{1}>4 K_{1}$, then we obtain from the Lyapounov structure (1.20) that there exists $T_{1}=T_{1}\left(R_{1}, d_{1}\right)$ such that

$$
v_{1}\left(\left[-d_{1}, d_{1}\right]\right) \geqslant \frac{1}{2} \text {. }
$$

Combining (1.39), (1.40) and (1.41) gives

$$
\mathbb{P}\left(Z_{1}\left(T_{1}\right)=Z_{2}\left(T_{1}\right),\left|Z_{1}\left(T_{1}\right)\right| \leqslant d_{1}\right) \geqslant C\left(p, d_{1}, T_{1}, R_{1}\right)>0 .
$$

Note that

$$
\begin{aligned}
& \mathbb{P}\left(Z_{1}\left(T_{1}\right)=Z_{2}\left(T_{1}\right),\left|u_{i}\left(T_{1}\right)\right| \leqslant d_{1}+d_{2}, i=1,2\right) \\
& \quad \geqslant \mathbb{P}\left(Z_{1}\left(T_{1}\right)=Z_{2}\left(T_{1}\right),\left|Z_{1}\left(T_{1}\right)\right| \leqslant d_{1}\right)-\sum_{i=1}^{2} \mathbb{P}\left(\left|u_{i}\left(T_{1}\right)\right| \geqslant d_{2}\right) .
\end{aligned}
$$

Using the Lyapounov structure (1.20), we obtain that

$$
\mathbb{P}\left(\left|u_{i}\left(T_{1}\right)\right| \geqslant d_{2}\right) \leqslant \frac{R_{1}+K_{1}}{d_{2}^{2}} .
$$

Combining (1.42), (1.43) and (1.44), we can choose $d_{2}$ sufficiently high such that, by setting $d^{*}=d_{1}+d_{2}, d_{0}=\left(2 d^{*}\right)^{2}$ and $\hat{p}=\frac{1}{2} C\left(2, d_{1}, T_{1}, R_{1}\right),(1.32)$ holds.

\subsection{Abstract result}

We now state and prove an abstract result which allows to reduce the proof of exponential convergence to equilibrium to the verification of some conditions, as was done in the previous section.

This result is closely related to the abstract result of [17]. Our proof has some similarity with the one in the reference but, in fact, is closer to arguments used in [22]. Our abstract result could be used in articles [11,13-15] and [17] to conclude.

In fact, in [17] a family $\left(r_{k}, s_{k}\right)$ of subprobability are used, whereas in [11,15] a family of subsets $Q(l, k)$ are introduced. Here, we use a random integer valued process $l_{0}(k)$. The three points of view are equivalent, the correspondence is given by

$$
s_{k+1}=\mathbb{P}\left(\left\{l_{0}(k+1)=1\right\} \cap \cdot\right), \quad r_{k+1}=\mathbb{P}\left(\left\{l_{0}(k+1)=1\right\}^{c} \cap \cdot\right),
$$

and

$$
Q(l, k)=\left\{l_{0}(k)=l\right\} .
$$

The result has already been applied in Section 1.2, the function used below is

$$
\mathcal{H}\left(u_{0}\right)=\left|u_{0}\right|^{2}
$$

in this example. In fact, in most of the application and in particular for the CGL equation in the first case treated below, $\mathcal{H}$ will be the square of the norm. We are concerned with $v\left(\cdot,\left(u_{0}, W_{0}\right)\right)=\left(u\left(\cdot, u_{0}\right), W\left(\cdot, W_{0}\right)\right)$, a couple of strongly Markovian process defined on polish spaces $\left(E, d_{E}\right)$ and $\left(F, d_{F}\right)$. We denote by $\left(\mathcal{P}_{t}\right)_{t \in I}$ the Markovian transition semigroup of $u$, where $I=\mathbb{R}^{+}$or $T \mathbb{N}=\{k T, k \in \mathbb{N}\}$.

We consider for any initial conditions $\left(v_{0}^{1}, v_{0}^{2}\right)$ a coupling $\left(v_{1}, v_{2}\right)$ of $\left(\mathcal{D}\left(v\left(\cdot, v_{0}^{1}\right)\right), \mathcal{D}\left(v\left(\cdot, v_{0}^{2}\right)\right)\right)$ and a random integer valued process $l_{0}: \mathbb{N} \rightarrow \mathbb{N} \cup\{\infty\}$ which has the following properties

$$
\left\{\begin{array}{l}
l_{0}(k+1)=l \text { implies } l_{0}(k)=l, \text { for any } l \leqslant k, \\
l_{0}(k) \in\{0,1,2, \ldots, k\} \cup\{\infty\}, \\
l_{0}(k) \text { depends only of }\left.v_{1}\right|_{[0, k T]} \text { and }\left.v_{2}\right|_{[0, k T]}, \\
l_{0}(k)=k \text { implies } \mathcal{H}_{k} \leqslant d_{0},
\end{array}\right.
$$


where

$$
\mathcal{H}_{k}=\mathcal{H}\left(u_{1}(k T)\right)+\mathcal{H}\left(u_{2}(k T)\right), \quad \mathcal{H}: E \rightarrow \mathbb{R}^{+} .
$$

We write $v_{i}=\left(u_{i}, W_{i}\right)$. From now on we say that $\left(v_{1}, v_{2}\right)$ are coupled at $k T$ if $l_{0}(k) \leqslant k$, in other words if $l_{0}(k) \neq \infty$.

Now we see four conditions on the coupling. The first condition states that when $\left(v_{1}, v_{2}\right)$ have been coupled for a long time then the probability that $\left(u_{1}, u_{2}\right)$ are close is high.

$$
\left\{\begin{array}{l}
\text { There exist } c_{0} \text { and } \alpha_{0}>0 \text { such that } \\
\mathbb{P}\left(d_{E}\left(u_{1}(t), u_{2}(t)\right)>c_{0} \mathrm{e}^{-\alpha_{0}(t-l T)} \text { and } l_{0}(k)=l\right) \leqslant c_{0} \mathrm{e}^{-\alpha_{0}(t-l T)},
\end{array}\right.
$$

for any $t \in[l T, k T] \cap I$.

The following property states that the probability that two solutions decouples at $k T$ is very small

$$
\left\{\begin{array}{l}
\text { There exist }\left(p_{k}\right)_{k \in \mathbb{N}}, c_{1}>0, \alpha_{1}>0 \text { such that } \\
\mathbb{P}\left(l_{0}(k+1)=l \mid l_{0}(k)=l\right) \geqslant p_{k-l}, \text { for any } l \leqslant k, \\
1-p_{k} \leqslant c_{1} \mathrm{e}^{-\alpha_{1} k T}, p_{k}>0 \text { for any } k \in \mathbb{N} .
\end{array}\right.
$$

Next condition states that, inside a ball, the probability that two solutions get coupled at $(k+1) T$ is uniformly bounded below.

$$
\left\{\begin{array}{l}
\text { There exist } p_{-1}>0, R_{0}>0 \text { such that } \\
\mathbb{P}\left(l_{0}(k+1)=k+1 \mid l_{0}(k)=\infty, \mathcal{H}_{k} \leqslant R_{0}\right) \geqslant p_{-1} .
\end{array}\right.
$$

The last ingredient is the so-called Lyapounov structure. It allows the control of the probability to enter the ball of radius $R_{0}$. It states that there exists $\gamma>1$, such that for any solution $v_{0}$

$$
\left\{\begin{array}{l}
\mathbb{E} \mathcal{H}\left(v\left(t, v_{0}\right)\right) \leqslant \mathrm{e}^{-\alpha_{3} t} \mathcal{H}\left(v_{0}\right)+\frac{K_{1}}{2}, \\
\mathbb{E}\left(\mathcal{H}\left(v\left(\tau^{\prime}, v_{0}\right)\right)^{\gamma} 1_{\tau^{\prime}<\infty}\right) \leqslant K^{\prime}\left(\mathcal{H}\left(v_{0}\right)+1\right)^{\gamma}, \\
\text { for any stopping times } \tau^{\prime} \text { taking value in }\{k T, k \in \mathbb{N}\} \cup\{\infty\} .
\end{array}\right.
$$

The process $V=\left(v_{1}, v_{2}\right)$ is said to be $l_{0}$-Markovian if the laws of $V(k T+\cdot)$ and of $l_{0}(k+\cdot)-k$ on $\left\{l_{0}(k) \in\{k, \infty\}\right\}$ conditioned by $\mathcal{F}_{k T}$ only depend on $V(k T)$ and are equal to the laws of $V(\cdot, V(k T))$ and $l_{0}$, respectively.

Notice that in the example of the previous section or in the CGL case below, the process $\left(u_{i}, W_{i}\right)_{i=1,2}$ is $l_{0^{-}}$ Markovian but not Markovian. However, in both cases, if we choose $d_{0}=R_{0}$, we can modify the coupling such that the couple is Markovian at discrete times $T \mathbb{N}=\{k T, k \in \mathbb{N}\}$. But it does not seem to be possible to modify the coupling to become Markovian at any times.

Theorem 1.8. Assume that (1.45)-(1.48) and (1.49) hold with $R_{0}>4 K_{1}$ and $R_{0} \geqslant d_{0}$ and that $V=\left(v_{1}, v_{2}\right)$ is $l_{0^{-}}$ Markovian. Then there exist $\alpha_{4}>0$ and $c_{4}>0$ such that

$$
\mathbb{P}\left(d_{E}\left(u_{1}(t), u_{2}(t)\right)>c_{3} \mathrm{e}^{-\alpha_{4} t}\right) \leqslant c_{3} \mathrm{e}^{-\alpha_{4} t}\left(1+\mathcal{H}\left(u_{0}^{1}\right)+\mathcal{H}\left(u_{0}^{2}\right)\right) .
$$

Moreover there exists a unique stationary probability measure $v$ of $\left(\mathcal{P}_{t}\right)_{t \in I}$ on E. It satisfies,

$$
\int_{E} \mathcal{H}(u) \mathrm{d} v(u) \leqslant \frac{K_{1}}{2},
$$

and there exists $c_{4}>0$ such that for any $\mu \in \mathcal{P}(E)$

$$
\left|\mathcal{P}_{t}^{*} \mu-v\right|_{\operatorname{Lip}_{b}(E)}^{*} \leqslant c_{4} \mathrm{e}^{-\alpha_{4} t}\left(1+\int_{E} \mathcal{H}(u) \mathrm{d} \mu(u)\right) .
$$

Proposition 1.6 is an easy consequence of Theorem 1.8. Actually (1.45) is clear and (1.46) and (1.49) are consequence of (1.17) and (1.20) if $R_{0} \geqslant d_{0}$. Finally, since, for any $\left(R_{0}, d_{0}, T\right)$ sufficiently high, there exists a coupling such that (1.18) and (1.19) hold, we can choose $\left(R_{0}, d_{0}, T\right)$ such that all our assumptions are true. 
Remark 1.9. Inequality (1.52) means that for any $f \in \operatorname{Lip}_{b}(E)$ and any $u_{0} \in E$

$$
\left|\mathbb{E} f\left(u\left(t, u_{0}\right)\right)-\int_{E} f(u) \mathrm{d} v(u)\right| \leqslant c_{4}|f|_{\operatorname{Lip}_{b}(E)} \mathrm{e}^{-\alpha_{4} t}\left(1+\mathcal{H}\left(u_{0}\right)\right) \text {. }
$$

\subsection{Proof of Theorem 1.8}

Reformulation of the problem. We rewrite our problem in the form on a exponential estimate.

As in the example, it is sufficient to establish (1.50). Then (1.51) is a simple consequence of (1.49) and (1.52) follows from (1.16). Assume that $t>8 T$. We denote by $k$ the unique integer such that $t \in(2(k-1) T, 2 k T]$. Notice that

$$
\begin{aligned}
& \mathbb{P}\left(d_{E}\left(u_{1}(t), u_{2}(t)\right)>c_{0} \mathrm{e}^{-\alpha_{0}(t-(k-1) T)}\right) \\
& \quad \leqslant \mathbb{P}\left(l_{0}(2 k) \geqslant k\right)+\mathbb{P}\left(d_{E}\left(u_{1}(t), u_{2}(t)\right)>c_{0} \mathrm{e}^{-\alpha_{0}(t-(k-1) T)} \text { and } l_{0}(2 k)<k\right) .
\end{aligned}
$$

Thus applying (1.46), using $2(t-(k-1) T)>t$, it follows

$$
\mathbb{P}\left(d_{E}\left(u_{1}(t), u_{2}(t)\right)>c_{0} \exp \left(-\frac{\alpha_{0}}{2} t\right)\right) \leqslant \mathbb{P}\left(l_{0}(2 k) \geqslant k\right)+c_{0} \exp \left(-\frac{\alpha_{0}}{2} t\right) .
$$

In order to estimate $\mathbb{P}\left(l_{0}(2 k) \geqslant k\right)$, we introduce the following notation

$$
l_{0}(\infty)=\lim \sup l_{0} .
$$

Taking into account (1.45), we obtain that for $l<\infty$

$$
\left\{l_{0}(\infty)=l\right\}=\left\{l_{0}(k)=l, \text { for any } k \geqslant l\right\} .
$$

We deduce

$$
\mathbb{P}\left(l_{0}(2 k) \geqslant k\right) \leqslant \mathbb{P}\left(l_{0}(\infty) \geqslant k\right) .
$$

Taking into account (1.53), (1.54) and using a Chebyshev inequality, it is sufficient to obtain that there exist $c_{5}>0$ and $\delta>0$ such that

$$
\mathbb{E}\left(\exp \left(\delta l_{0}(\infty)\right)\right) \leqslant c_{5}\left(1+\mathcal{H}\left(u_{0}^{1}\right)+\mathcal{H}\left(u_{0}^{2}\right)\right) .
$$

Then (1.50) follows with

$$
\alpha_{4}=\min \left\{\frac{\alpha_{0}}{2}, \frac{\delta}{2 T}\right\} .
$$

Definition of a sequence of stopping times. Using the Lyapounov structure (1.49), we prove at the end this subsection that there exist $\delta_{0}>0$ and $c_{6}>0$ such that

$$
\mathbb{E}\left(\exp \left(\delta_{0} \tau\right)\right) \leqslant c_{6}\left(1+\mathcal{H}\left(u_{0}^{1}\right)+\mathcal{H}\left(u_{0}^{2}\right)\right),
$$

where

$$
\tau=\min \left\{t \in T \mathbb{N} \mid \mathcal{H}\left(u_{1}(t)\right)+\mathcal{H}\left(u_{2}(t)\right) \leqslant R_{0}\right\} .
$$

We set

$$
\hat{\sigma}=\min \left\{k \in \mathbb{N}^{*} \mid l_{0}(k)>1\right\}, \quad \sigma=\hat{\sigma} T .
$$

Clearly $\hat{\sigma}=1$ if the two solutions do not get coupled at time 0 or $T$. Otherwise, they get coupled at 0 or $T$ and remain coupled until $\sigma$.

Let us assume for the moment that if $\mathcal{H}_{0} \leqslant R_{0}$, then

$$
\left\{\begin{array}{l}
\mathbb{E}\left(\exp \left(\delta_{1} \sigma\right) 1_{\sigma<\infty}\right) \leqslant c_{7} \\
\mathbb{P}(\sigma=\infty) \geqslant p_{\infty}>0
\end{array}\right.
$$

The proof is given after the proof of (1.56) at the end of this subsection. 
Now we build a sequence of stopping times

$$
\begin{aligned}
& \tau_{0}=\tau, \\
& \hat{\sigma}_{k+1}=\min \left\{l \in \mathbb{N}^{*} \mid l T>\tau_{k} \text { and } l_{0}(l) T>\tau_{k}+T\right\}, \quad \sigma_{k+1}=\hat{\sigma}_{k+1} \times T, \\
& \tau_{k+1}=\sigma_{k+1}+\tau o \theta_{\sigma_{k+1}},
\end{aligned}
$$

where $\left(\theta_{t}\right)_{t}$ is the shift operator. The idea is the following. We wait the time $\tau_{k}$ to enter the ball of radius $R_{0}$. Then, if we do not start coupling at time $\tau_{k}$, we try to couple at time $\tau_{k}+T$. If we fail to start coupling at time $\tau_{k}$ or $\tau_{k}+T$ we set $\sigma_{k}=\tau_{k}+T$ else we set $\sigma_{k}$ the time the coupling fails ( $\sigma_{k}=\infty$ if the coupling never fails). Then if $\sigma_{k}<\infty$, we retry to enter the ball of radius $R_{0}$. The fact that $R_{0} \geqslant d_{0}$ implies that $l_{0}\left(\tau_{k}\right) \in\left\{\tau_{k}, \infty\right\}$.

The idea of the $l_{0}$-Markovian property is the following. Since $l_{0}\left(\tau_{k}\right) \in\left\{\tau_{k}, \infty\right\}$ and $l_{0}\left(\sigma_{k}\right) \in\left\{\sigma_{k}, \infty\right\}$, when these stopping times are finite and since these stopping times are taking value in $T \mathbb{N} \cup\{\infty\}$, then the $l_{0}$-Markovian property implies the strong Markovian property when conditioning with respect to $\mathcal{F}_{\tau_{k}}$ or $\mathcal{F}_{\sigma_{k}}$. Moreover, we infer from the $l_{0}$-Markovian property of $V$ that

$$
\sigma_{k+1}=\tau_{k}+\sigma o \theta_{\tau_{k}},
$$

which implies

$$
\tau_{k+1}=\tau_{k}+\rho o \theta_{\tau_{k}}, \quad \text { where } \rho=\sigma+\tau o \theta_{\sigma} .
$$

Exponential estimate on $\rho$. Before concluding, we establish that there exist $K$ such that for any $V_{0}$ such that $\mathcal{H}_{0} \leqslant R_{0}$ and for any $\delta_{2} \leqslant \frac{1}{\gamma^{\prime}}\left(\delta_{0} \wedge \delta_{1}\right)$

$$
\mathbb{E}_{V_{0}}\left(\mathrm{e}^{\delta_{2} \rho} 1_{\rho<\infty}\right) \leqslant K \text {. }
$$

Notice that for any $V_{0}$ such that $\mathcal{H}_{0} \leqslant R_{0}$,

$$
\mathbb{E}_{V_{0}}\left(\mathrm{e}^{\delta_{2} \rho} 1_{\rho<\infty}\right)=\mathbb{E}_{V_{0}}\left(\mathrm{e}^{\delta_{2} \sigma} 1_{\sigma<\infty} \mathbb{E}\left(\mathrm{e}^{\delta_{2} \tau o \theta_{\sigma}} 1_{\tau o \theta_{\sigma}<\infty} \mid \mathcal{F}_{\sigma}\right)\right) .
$$

Applying the $l_{0}$-Markovian property and (1.56), we obtain

$$
\mathbb{E}\left(\mathrm{e}^{\delta_{2} \tau o \theta_{\sigma}} 1_{\tau o \theta_{\sigma}<\infty} \mid \mathcal{F}_{\sigma}\right) \leqslant c_{6}\left(1+\mathcal{H}\left(u_{1}(\sigma)\right)+\mathcal{H}\left(u_{2}(\sigma)\right)\right),
$$

which implies

$$
\mathbb{E}_{V_{0}}\left(\mathrm{e}^{\delta_{2} \rho} 1_{\rho<\infty}\right) \leqslant c_{6} \mathbb{E}_{V_{0}}\left(\mathrm{e}^{\delta_{2} \sigma} 1_{\sigma<\infty}\left(1+\mathcal{H}\left(u_{1}(\sigma)\right)+\mathcal{H}\left(u_{2}(\sigma)\right)\right)\right) .
$$

An Hölder inequality gives

$$
\mathbb{E}_{V_{0}}\left(\mathrm{e}^{\delta_{2} \rho} 1_{\rho<\infty}\right) \leqslant c_{6}\left(\mathbb{E}_{V_{0}} \mathrm{e}^{\gamma^{\prime} \delta_{2} \sigma} 1_{\sigma<\infty}\right)^{1 / \gamma^{\prime}}\left(\mathbb{E}_{V_{0}}\left(1+\mathcal{H}\left(u_{1}(\sigma)\right)+\mathcal{H}\left(u_{2}(\sigma)\right)\right)^{\gamma}\right)^{1 / \gamma} .
$$

Applying the Lyapounov structure (1.49) and (1.57), we obtain (1.58).

Conclusion. We remark that

$$
\mathbb{E}\left(\mathrm{e}^{\delta_{2} \tau_{k+1}} 1_{\tau_{k+1}<\infty}\right)=\mathbb{E}\left(\mathrm{e}^{\delta_{2} \tau_{k}} 1_{\tau_{k}<\infty} \mathbb{E}\left(\mathrm{e}^{\delta_{2} \rho o \theta_{\tau_{k}}} 1_{\rho o \theta_{\tau_{k}}<\infty} \mid \mathcal{F}_{\tau_{k}}\right)\right) .
$$

Applying again the $l_{0}$-Markov property of $V$

$$
\mathbb{E}\left(\mathrm{e}^{\delta_{2} \tau_{k+1}} 1_{\tau_{k+1}<\infty}\right)=\mathbb{E}\left(\mathrm{e}^{\delta_{2} \tau_{k}} 1_{\tau_{k}<\infty} \mathbb{E}_{V\left(\tau_{k}\right)}\left(\mathrm{e}^{\delta_{2} \rho} 1_{\rho<\infty}\right)\right)
$$

Iterating (1.59) by using (1.58) and (1.56), we obtain

$$
\mathbb{E} \mathrm{e}^{\delta_{2} \tau_{n}} 1_{\tau_{n}<\infty} \leqslant c_{6} K^{n}\left(1+\mathcal{H}\left(u_{0}^{1}\right)+\mathcal{H}\left(u_{0}^{2}\right)\right) .
$$

Using the second inequality of (1.57) and that $\tau<\infty$, we obtain from the $l_{0}$-Markov property that

$$
\mathbb{P}\left(k_{0}>n\right) \leqslant\left(1-p_{\infty}\right)^{n},
$$

where

$$
k_{0}=\inf \left\{k \in \mathbb{N} \mid \sigma_{k+1}=\infty\right\} .
$$


Then we obtain that $k_{0}<\infty$ almost surely and that

$$
l_{0}(\infty) \in\left\{\tau_{k_{0}}, \tau_{k_{0}}+1\right\} .
$$

Therefore $l_{0}(\infty)<\infty$ almost surely and

$$
\mathbb{E} \exp \left(\frac{\delta_{2}}{p} l_{0}(\infty)\right) \leqslant \sum_{n=1}^{\infty} \mathbb{E} \mathrm{e}^{\left(\delta_{2} / p\right)\left(\tau_{n}+1\right)} 1_{k_{0}=n},
$$

which implies, by applying a Hölder inequality,

$$
\mathbb{E} \exp \left(\frac{\delta_{2}}{p} l_{0}(\infty)\right) \leqslant \mathrm{e}^{\delta_{2} / p} \sum_{n=1}^{\infty}\left(\mathbb{E} \mathrm{e}^{\delta_{2} \tau_{n}} 1_{\tau_{n} \leqslant \infty}\right)^{1 / p}\left(\mathbb{P}\left(k_{0}=n\right)\right)^{1 / p^{\prime}} .
$$

Applying (1.60) and (1.61), we obtain

$$
\mathbb{E} \exp \left(\frac{\delta_{2}}{p} l_{0}(\infty)\right) \leqslant c_{6} \mathrm{e}^{\delta_{2} / p}\left(\sum_{n=1}^{\infty}\left(K^{1 / p}\left(1-p_{\infty}\right)^{1 / p^{\prime}}\right)^{n}\right)\left(1+\mathcal{H}\left(u_{0}^{1}\right)+\mathcal{H}\left(u_{0}^{2}\right)\right)^{1 / p} .
$$

Choosing $p$ such that $K^{1 / p}\left(1-p_{\infty}\right)^{1 / p^{\prime}}<1$ and setting $\delta=\delta_{2} / p$, we obtain (1.55)

Proof of (1.56). Let $N$ be an integer such that

$$
\mathrm{e}^{-\alpha_{3} N T} \leqslant \frac{1}{8}
$$

We fix $i \in\{1,2\}$ and set

$$
B_{k}=\left\{\mathcal{H}\left(u_{i}(j N T)\right) \geqslant 2 K_{1}, \text { for any } j \leqslant k\right\}, \quad C_{k}=\left\{\mathcal{H}\left(u_{i}(k N T)\right) \geqslant 2 K_{1}\right\} .
$$

Combining the Markov property of $u_{i}$ and the Lyapounov structure (1.49), we obtain

$$
\mathbb{E}\left(\mathcal{H}\left(u_{i}((k+1) N T)\right) \mid \mathcal{F}_{k N T}\right) \leqslant \frac{1}{4} \mathcal{H}\left(u_{i}(k N T)\right)+\frac{K_{1}}{2} .
$$

Hence, applying a Chebyshev inequality, it follows that

$$
\mathbb{P}\left(C_{k+1} \mid \mathcal{F}_{k N T}\right) \leqslant \frac{1}{8 K_{1}} \mathcal{H}\left(u_{i}(k N T)\right)+\frac{1}{4} .
$$

Integrating (1.62), (1.63) over $B_{k}$, we obtain that

$$
\left(\begin{array}{c}
\mathbb{E}\left(\mathcal{H}\left(u_{i}((k+1) N T)\right) 1_{B_{k+1}}\right) \\
\mathbb{P}\left(B_{k+1}\right)
\end{array}\right) \leqslant A\left(\begin{array}{c}
\mathbb{E}\left(\mathcal{H}\left(u_{i}(k N T)\right) 1_{B_{k}}\right) \\
\mathbb{P}\left(B_{k}\right)
\end{array}\right)
$$

where

$$
A=\left(\begin{array}{cc}
\frac{1}{4} & \frac{K_{1}}{2} \\
\frac{1}{8 K_{1}} & \frac{1}{4}
\end{array}\right)
$$

Since the eigenvalues of $A$ are 0 and $\frac{1}{2}$, we obtain that

$$
\mathbb{P}\left(B_{k}\right) \leqslant \frac{2}{K_{1}}\left(\frac{1}{2}\right)^{k}\left(1+\mathcal{H}\left(u_{0}^{i}\right)\right) .
$$

It follows from $R_{0} \geqslant 4 K_{1}$ that

$$
\mathbb{P}(\tau>k T) \leqslant c \exp \left(-\frac{k}{N} \ln 2\right)\left(1+\mathcal{H}\left(u_{0}^{i}\right)\right) .
$$

Hence, taking $\delta_{0}<\alpha_{3} / 3$, we have established (1.56). 
Proof of (1.57). Now we establish (1.57). There are two cases. The first case is $l_{0}(0)=0$. Then, applying (1.47), we obtain that

$$
\mathbb{P}(\sigma=\infty) \geqslant \prod_{k=0}^{\infty} \mathbb{P}\left(l_{0}(k+1)=0 \mid l_{0}(k)=0\right) \geqslant \prod_{k=0}^{\infty} p_{k} .
$$

The second case is $l_{0}(0)=\infty$. Then

$$
\mathbb{P}(\sigma=\infty) \geqslant \mathbb{P}\left(l_{0}(1)=1\right) \prod_{k=1}^{\infty} \mathbb{P}\left(l_{0}(k+1)=1 \mid l_{0}(k)=1\right) .
$$

Since $\mathcal{H}_{0} \leqslant R_{0}$, then applying (1.47) and (1.48)

$$
\mathbb{P}(\sigma=\infty) \geqslant \prod_{k=-1}^{\infty} p_{k}
$$

Since $p_{k}>0$ and $1-p_{k}$ exponentially decreases, then the product converges and in the two cases

$$
\mathbb{P}(\sigma=\infty) \geqslant p_{\infty}=\prod_{k=-1}^{\infty} p_{k}>0 .
$$

Notice that (1.47) implies

$$
\mathbb{P}(\sigma=n) \leqslant \mathbb{P}\left(l_{0}(n+1) \neq n \mid l_{0}(n)=0\right)+\mathbb{P}\left(l_{0}(n+1) \neq n \mid l_{0}(n)=1\right) \leqslant 2 c_{1} \mathrm{e}^{-\alpha_{1}(n-1) T},
$$

which gives the first inequality of (1.56) and allows to conclude

\section{Properties of the CGL equation}

We are concerned with the stochastic Complex Ginzburg-Landau (CGL) equations with Dirichlet boundary conditions:

$$
\left\{\begin{array}{l}
\frac{\mathrm{d} u}{\mathrm{~d} t}-(\varepsilon+\mathrm{i}) \Delta u+(\eta+\lambda \mathrm{i})|u|^{2 \sigma} u=b(u) \frac{\mathrm{d} W}{\mathrm{~d} t}+f, \\
u(t, x)=0, \quad \text { for } x \in \delta D \\
u(0, x)=u_{0}(x),
\end{array}\right.
$$

where $\varepsilon>0, \eta>0, \lambda \in\{-1,1\}$ and where $D$ is an open bounded set of $\mathbb{R}^{d}$ with sufficiently regular boundary or $D=[0,1]^{d}$. Also $f$ is the deterministic part of the forcing term. For simplicity in the redaction, we consider the case $f=0$. The generalisation to a square integrable $f$ is easy. We say that it is the defocussing or the focusing equation when $\lambda$ is equal to 1 or -1 , respectively.

We set

$$
A=-\Delta, \quad D(A)=H_{0}^{1}(D) \cap H^{2}(D) .
$$

Now we can write problem (2.1) in the form

$$
\begin{aligned}
& \frac{\mathrm{d} u}{\mathrm{~d} t}+(\varepsilon+\mathrm{i}) A u+(\eta+\lambda \mathrm{i})|u|^{2 \sigma} u=b(u) \frac{\mathrm{d} W}{\mathrm{~d} t}, \\
& u(0)=u_{0},
\end{aligned}
$$

where $W$ is a cylindrical Wiener process of $L^{2}(D)$.

The aim of this section is to prove some properties which will be used in Section 3 to build a coupling such that the assumptions of Theorem 1.8 are true. 


\subsection{Notations and main result}

We consider $\left(e_{n}, \mu_{n}\right)_{n \in \mathbb{N}^{*}}$ the couples of eigenvalues and eigenvectors of $A\left(A e_{n}=\mu_{n}\right)$ such that $\left(e_{n}\right)_{n}$ is an Hilbertian basis of $L^{2}(D)$ and such that $\left(\mu_{n}\right)_{n}$ is an increasing sequence. We denote by $P_{N}$ and $Q_{N}$ the orthogonal projection in $L^{2}(D)$ on the space $S p\left(e_{k}\right)_{1 \leqslant n}$ and on its complementary, respectively.

The first condition is a condition on the smoothness of the noise and a condition ensuring existence and uniqueness of solutions.

We will sometimes consider the $L^{2}(D)$ subcritical condition:

H1 We assume that $0<\sigma<\frac{2}{d} \wedge \frac{3}{2}$. Moreover $u_{0} \in L^{2}(D)$ and $b$ is bounded Lipschitz

$$
b: L^{2}(D) \rightarrow \mathcal{L}_{2}\left(L^{2}(D), H^{2}(D)\right) .
$$

We also consider the $H^{1}(D)$ subcritical condition when the equation is defocussing.

$\mathrm{H}^{\prime}$ If $d \leqslant 2$ we assume that $\sigma>0$. If $d>2$, we assume that $0<\sigma<\frac{2}{d-2}$. Moreover $\lambda=1, u_{0} \in H^{1}(D)$ and $b$ is bounded Lipschitz

$$
b: L^{2}(D) \rightarrow \mathcal{L}_{2}\left(L^{2}(D), H^{2}(D)\right) .
$$

We set, for $s \leqslant 2$,

$$
B_{s}=\sup _{u}|b(u)|_{\mathcal{L}_{2}\left(L^{2}(D), H^{s}(D)\right)}^{2} .
$$

The second assumption means that $b$ only depends on its low modes.

$\mathrm{H} 2$ There exists $N_{1}$ such that

$$
b(u)=b\left(P_{N_{1}} u\right) .
$$

The third condition is a structure condition on $b$. It is a slight generalisation of the usual assumption that $b(u)$ is diagonal in the basis $\left(e_{n}\right)_{n}$.

H3 There exists $N \geqslant N_{1}$, such that for any $u$,

$$
P_{N} b(u) Q_{N}=0, \quad Q_{N} b(u) P_{N}=0 .
$$

Moreover $P_{N} b(u) P_{N}$ is invertible on $P_{N} H$ and

$$
\sup _{u}\left|\left(P_{N} b(u) P_{N}\right)^{-1}\right|<\infty .
$$

In this section, we define by $|\cdot|,|\cdot|_{p},\|\cdot\|$ and $\|\cdot\|_{s}$ the norm of $L^{2}(D), L^{p}(D), H^{1}(D)$ and $H^{s}(D)$.

The Lyapounov structures are defined by

$$
\begin{aligned}
& \mathcal{H}^{L^{2}}=|\cdot|^{2}, \\
& \mathcal{H}^{H^{1}}=\frac{1}{2}\|\cdot\|^{2}+\frac{1}{2 \sigma+2}|\cdot|_{2 \sigma+2}^{2 \sigma+2} .
\end{aligned}
$$

The energies are defined by

$$
E_{u}^{L^{2}}(t, T)=|u(t)|^{2}+\varepsilon \int_{T}^{t}\|u(s)\|^{2} \mathrm{~d} s,
$$

and 


$$
E_{u}^{H^{1}}(t, T)=\mathcal{H}^{H^{1}}(u(t))+\frac{\varepsilon}{2} \int_{T}^{t}\|u(s)\|_{2}^{2} \mathrm{~d} s+\frac{\eta}{2} \int_{T}^{t}|u(s)|_{4 \sigma+2}^{4 \sigma+2} \mathrm{~d} s+(\eta+\varepsilon) \int_{T}^{t} \int_{D}|u(s, x)|^{2 \sigma}|\nabla u(s, x)|^{2} \mathrm{~d} x \mathrm{~d} s .
$$

When $T=0$, we simply write $E_{u}(t)=E_{u}(t, 0)$.

The first case is the $L^{2}$-subcritical focusing or defocussing CGL equation with initial condition in $L^{2}(D)$ :

\section{Case 1.}

- H1, H2 and H3 hold,

- $\lambda \in\{-1,1\}, H=L^{2}(D)$,

$-\mathcal{H}=\mathcal{H}^{L^{2}}=|\cdot|_{L^{2}(D)}^{2}, E_{u}=E_{u}^{L^{2}}$.

The second case is the $H^{1}$-subcritical defocussing CGL equation with initial condition in $H^{1}(D)$.

\section{Case 2.}

- H1', $\mathrm{H} 2$ and $\mathrm{H} 3$ hold,

$-\lambda=1, H=H^{1}(D)$,

$-\mathcal{H}=\mathcal{H}^{H^{1}}=\frac{1}{2}\|\cdot\|_{H^{1}(D)}^{2}+\frac{1}{2 \sigma+2}|\cdot|_{L^{2 \sigma+2}(D)}^{2 \sigma+2}, E_{u}=E_{u}^{H^{1}}$.

When it is not precised, the results stated are true in both cases. It is well known that we have existence and uniqueness of the solutions in both cases and that the solutions are strongly Markov process. We denote by $\left(\mathcal{P}_{t}\right)_{t \in \mathbb{R}^{+}}$ the Markov transition semi-group associated to the solutions of (2.2).

The aim of this article is to establish the following result

Theorem 2.1 ((Main theorem)). There exists $N_{0}\left(B_{2}, \eta, \varepsilon, \sigma, D\right)$ such that if $N \geqslant N_{0}$, then in cases 1 and 2 , there exists a unique stationary probability measure $v$ of $\left(\mathcal{P}_{t}\right)_{t \in \mathbb{R}^{+}}$on $L^{2}(D)$. Moreover, $v$ satisfies

$$
\int_{H}\|u\|_{H^{2}(D)}^{2} \mathrm{~d} v(u)<\infty
$$

and for any $s \in[0,2)$, there exists $C_{s}>0$ and $\alpha_{s}$ such that for any $\mu \in \mathcal{P}(H)$

$$
\left|\mathcal{P}_{t}^{*} \mu-v\right|_{\operatorname{Lip}_{b}\left(H^{s}(D)\right)}^{*} \leqslant C_{s} \mathrm{e}^{-\alpha_{s} t}\left(1+\int_{H}|u|_{L^{2}(D)}^{2} \mathrm{~d} \mu(u)\right) .
$$

Furthermore, if $(u, W)$ is a weak solution of (2.2), (2.3), with $u_{0}$ taking value in $L^{2}(D)$ then for any $f \in \operatorname{Lip}_{b}\left(H^{s}(D)\right)$

$$
\left|\mathbb{E} f(u(t))-\int_{H} f(u) \mathrm{d} v(u)\right| \leqslant C_{s}|f|_{\operatorname{Lip}_{b}\left(H^{s}(D)\right)} \mathrm{e}^{-\alpha_{s} t}\left(1+\mathbb{E}\left|u_{0}\right|_{L^{2}(D)}^{2}\right) .
$$

Remark 2.2. In case 1, (2.5) is equivalent to (2.6). But in case 2, the Markovian transition semi-group make sense only if $u_{0}$ is taking value in $H=H^{1}(D)$ because strong existence and weak uniqueness may cause problem when $u_{0} \in L^{2}(D)$. Hence (2.5) make sense only if $\mu \in \mathcal{P}\left(H^{1}(D)\right)$ which means that $u_{0} \in H^{1}(D)$.

Remark 2.3. Assume that $B_{s}<\infty$ for $s$ sufficiently high. Let $k$ be a positive integer such that

$$
k \leqslant 2 \sigma+2, \quad \text { if } \sigma \notin \mathbb{N}, \quad \text { and } \quad k \in \mathbb{N} \text { if } \sigma \in \mathbb{N} .
$$

Applying Remark 2.15 below and adapting the proof of Theorem 2.1, we obtain that (2.4) can be replaced by

$$
\int_{H}\|u\|_{H^{k}(D)}^{2} \mathrm{~d} v(u)<\infty,
$$


and (2.5) is true for any $s$ real number such that

$$
s<[2 \sigma+2], \quad \text { if } \sigma \notin \mathbb{N}, \quad \text { and } \quad s \in \mathbb{R} \quad \text { if } \sigma \in \mathbb{N},
$$

where $[\cdot]$ denote the integer part.

The condition on $k$ and $s$ comes from the lack of derivability of the non-linear part of the CGL equation. Assume that we replace $|u|^{2 \sigma} u$ by $g\left(|u|^{2}\right) u$ where

- $g$ is infinitely continuously differentiable,

$-g(x)=x^{\sigma}$ for $x \geqslant x_{0}$,

$-g$ is increasing and $g(0)=0$.

Hence Theorem 2.1, (2.7) and (2.5) are true for any $k$ and $s$.

\subsection{Properties of the solutions}

In this subsection, we state some properties proved in the next subsections. These are used in Section 3 to apply Theorem 1.8 in order to establish Theorem 2.1.

First, we recall the following result.

Proposition 2.4. In the two previous cases, there exists a measurable map

$$
\Phi: C\left((0, T) ; P_{N} H\right) \times C\left((0, T) ; Q_{N} H^{(d+1) / 2}(D)\right) \times H \rightarrow C\left((0, T) ; Q_{N} H\right),
$$

such that for any $(u, W)$ solution of (2.2) and (2.3)

$$
Q_{N} u=\Phi\left(P_{N} u, Q_{N} W, u_{0}\right) \quad \text { on }[0, T] .
$$

Moreover $\Phi$ is a non-anticipative functions of $\left(P_{N} u, Q_{N} W\right)$.

Proposition 2.4 can be proved by applying a fix point argument and by taking into account that the limit of a sequence of measurable maps is measurable.

We have the so-called Foias-Prodi estimates.

Proposition 2.5 ((Foias-Prodi estimate)). Let $u_{1}$ and $u_{2}$ be two solutions of the CGL system (2.2) associated with Wiener process $W_{1}$ and $W_{2}$ respectively. If

$$
P_{N} u_{1}(t)=P_{N} u_{2}(t), \quad Q_{N} W_{1}(t)=Q_{N} W_{2}(t), \quad \text { for } T_{0} \leqslant t \leqslant T,
$$

where $N$ is a non-negative integer, then

$$
|r(t)|_{H} \leqslant\left|r\left(T_{0}\right)\right|_{H} \exp \left(-\frac{\varepsilon \mu_{N+1}}{2}\left(t-T_{0}\right)+c_{1} \sum_{i=1}^{2} E_{u_{i}}\left(t, T_{0}\right)\right),
$$

where $r=u_{1}-u_{2}$ and $T_{0} \leqslant t \leqslant T$ and where $c_{1}>0$ only depends on $\varepsilon, \eta, \sigma, D$.

We deduce immediately a very useful corollary.

Corollary 2.6. For any $B$, there exists $N_{0}^{\prime}(B, \eta, \varepsilon, D, \sigma)$ such that under the assumptions of Proposition 2.5, under the assumption $N \geqslant N_{0}^{\prime}$ and under the assumption

$$
E_{u_{i}}\left(t, T_{0}\right) \leqslant \rho+B\left(t-T_{0}\right), \quad i=1,2,
$$

we obtain that

$$
|r(t)|_{H} \leqslant\left|r\left(T_{0}\right)\right|_{H} \exp \left(-2\left(t-T_{0}\right)+c_{1} \rho\right),
$$

where $c_{1}$ is the constant of Proposition 2.5. 
Then, by proving analogous result to the previous corollary, we obtain the Drift estimate which, in Section 3, will ensures the Novikov condition and will allow to apply the Girsanov formula.

Lemma 2.7 ((Drift estimate)). For any $B$, there exists $N_{0}^{\prime \prime}(B, \eta, \varepsilon, D, \sigma)$ such that for any $u_{1}, u_{2}$ solutions of the $C G L$ system (2.2) associated with $W_{1}$ and $W_{2}$ and for any $N>N_{0}^{\prime \prime}$

$$
\int_{T_{0}}^{\tau}\left|P_{N}\left(\left|u_{1}(s)\right|-\left|u_{2}(s)\right|\right)\right|^{2} \mathrm{~d} s \leqslant K_{N}|r(0)|^{2} \mathrm{e}^{c \rho-3 T_{0}},
$$

where $T>T_{0} \geqslant 0$ and $\rho, C, \alpha>0$, where $K_{N}, c$ only depend on $B, C, \alpha, \varepsilon, \eta, \sigma, D, N$ and where we have denoted by $\tau$ the value

$$
\tau=T_{0} \vee \inf \left(t \in[0, T] \mid \begin{array}{l|l}
E_{u_{1}}(t) \geqslant \rho+B t \text { or } E_{u_{2}}(t) \geqslant \rho+C\left(1+t^{\alpha}\right) \text { or } \\
P_{N} u_{1}(t) \neq P_{N} u_{2}(t) \text { or } Q_{N} W_{1}(t) \neq Q_{N} W_{2}(t)
\end{array}\right) .
$$

Now we set

$$
N_{0}=N_{0}^{\prime} \vee N_{0}^{\prime \prime} .
$$

In order to apply the previous lemmas and corollary, we establish the two following results.

Proposition 2.8 ((Exponential estimate for the growth of solution)). Assume that $u$ is a solution of (2.2), (2.3) associated with a Wiener process $W$. Then, for any $0 \leqslant T_{0}<T \leqslant \infty$

$$
\mathbb{P}\left(\sup _{t \in\left[T_{0}, T[\right.}\left(E_{u}(t)-B t\right) \geqslant \mathcal{H}\left(u_{0}\right)+\rho\right) \leqslant \mathrm{e}^{-\gamma_{0} \rho-3 T_{0}},
$$

where $B$ only depends on $B_{2}, \sigma, \eta, \varepsilon$.

Proposition 2.9. Assume that $u$ is a solution of (2.2), (2.3) associated with a Wiener process W. For any $u_{0}^{2}$, we define $\tilde{u}$ by

$$
\tilde{u}=P_{N} u+\phi\left(P_{N} u, Q_{N} W, u_{0}^{2}\right) .
$$

Then, there exists $\alpha \geqslant 1$ such that for any $N$, there exists $C_{N}$,

$$
\mathbb{P}\left(\sup _{t \in[0, T[}\left(E_{\tilde{u}}(t)-C_{N} t^{\alpha}\right) \geqslant C_{N}\left(1+\mathcal{H}\left(u_{0}\right)+\mathcal{H}\left(u_{0}^{2}\right)^{\alpha}+\rho\right)\right) \leqslant 2 \mathrm{e}^{-\gamma_{0} \rho},
$$

for any $0 \leqslant T \leqslant \infty$ and any $u_{0}^{2}$.

Let $u_{1}$ and $u_{2}$ be two solutions of (2.2) that correspond to deterministic initial value $u_{0}^{1}$ and $u_{0}^{2}$, respectively.

Lemma 2.10 ((The Lyapounov structure)). There exists $\alpha>0$ and $C_{k}>0$ such that for any $k$

$$
\mathbb{E} \mathcal{H}\left(u_{i}(t)\right)^{k} \leqslant \mathcal{H}\left(u_{0}^{i}\right)^{k} \mathrm{e}^{-\alpha k t}+\frac{C_{k}}{2},
$$

and for any stopping time $\tau$

$$
\mathbb{E} \mathcal{H}\left(u_{i}(\tau)\right)^{k} 1_{\tau<\infty} \leqslant \mathcal{H}\left(u_{0}^{i}\right)^{k}+C_{k} .
$$

Using Lemma 2.10 and Chebyshev's inequality, we obtain

Lemma 2.11. If $R_{0} \geqslant\left(\mathcal{H}\left(u_{0}^{1}\right)+\mathcal{H}\left(u_{0}^{2}\right)\right) \vee C_{1}$, then

$$
\mathbb{P}\left(\mathcal{H}\left(u_{1}(t)\right)+\mathcal{H}\left(u_{2}(t)\right) \geqslant 4 C_{1}\right) \leqslant \frac{1}{2},
$$

providing $t \geqslant \theta_{1}\left(R_{0}\right)=\frac{1}{\alpha} \ln \frac{R_{0}}{C_{1}}$. 
Then, in the second case, we control $\mathcal{H}(u(t))$ by $\left|u_{0}\right|^{2}$.

Proposition 2.12. It is assumed that $u$ is a solution of (2.2), (2.3) associated with a Wiener process W. Then, for any $T>0$

$$
\mathbb{E} \mathcal{H}(u(T)) \leqslant A+B T+\frac{C}{T}\left|u_{0}\right|^{2},
$$

where $A, B$ and $C$ only depends on $B_{2}, \sigma, \eta, \varepsilon$.

Now, we claim that in the two cases, we can control the norm of solutions in Sobolev spaces by the norm in $L^{2}$.

Proposition 2.13. Let $k$ be a positive integer less than 2. There exist $\gamma_{k}>1$ only depending on $k, \sigma$ and $d$ and $C_{k}>0$ and $c_{k}>0$ only depending on $k,\left(B_{s}\right)_{s}, \sigma, d, \varepsilon$ and $\eta$ such that for any $T>0$ and $t>0$

$$
\mathbb{E}\left(\|u(T+t)\|_{k}^{2}+\int_{T}^{T+t}\|u(s)\|_{k+1}^{2} \mathrm{~d} s\right)^{2 / \gamma_{k}} \leqslant c_{k} \frac{1}{T}\left|u_{0}\right|^{2}+C_{k}(1+T+t) .
$$

Hence, applying a Chebyshev inequality, we obtain

Corollary 2.14. Let $k$ be a positive integer less than 2 and $\delta>0$. There exist $\gamma>0$ only depending on $k, \sigma$ and $d$ and $C_{\delta}>0$ only depending on $\delta, k,\left(B_{s}\right)_{s}, \sigma, d, \varepsilon$ and $\eta$ such that for any $t>0$

$$
\mathbb{P}\left(\|u(t)\|_{k} \geqslant \mathrm{e}^{\delta t}\right) \leqslant C_{\delta} \mathrm{e}^{-\frac{\delta}{\gamma} t}\left(\left|u_{0}\right|^{2}+1\right) .
$$

Remark 2.15. Assume that $B_{s}<\infty$ for $s$ sufficiently high. The proof of Proposition 2.13 can be adapted to $k$ a positive integer such that

$$
k \leqslant 2 \sigma+2, \quad \text { if } \sigma \notin \mathbb{N}, \quad \text { and } \quad k \in \mathbb{N} \quad \text { if } \sigma \in \mathbb{N},
$$

and then Corollary 2.14 is true for such a $k$.

The condition on $k$ comes from the fact that $|\cdot|^{\sigma}$ is not $C^{\infty}$ on 0 . As in Remark 2.3 , if we replace $|\cdot|^{\sigma}$ by a nice function which coincides with $|\cdot|^{\sigma}$ on $\left[x_{0}, \infty\right)$, we can establish those results for any $k$.

\subsection{Foias-Prodi and Drift estimates}

The proofs in the first case are closely related to the proofs in the second case, but are simpler. That is the reason why we only give the proof in the second case.

Proof of Proposition 2.5 in the second case. We denote $u_{1}-u_{2}$ by $r$.

Step 1. This step is devoted to the proof of

$$
I=\left((\eta+\mathrm{i})\left(\left|u_{2}\right|^{2 \sigma} u_{2}-\left|u_{1}\right|^{2 \sigma} u_{1}\right), A r\right) \leqslant \frac{\varepsilon}{2}\|r\|_{2}^{2}+c\|r\|^{2} \sum_{i}\left|u_{i}\right|_{4 \sigma+2}^{4 \sigma+2} .
$$

We recall the following estimate

$$
\left.|| x\right|^{2 \sigma} x-|y|^{2 \sigma} y|\leqslant c| x-y \mid\left(|x|^{2 \sigma}+|y|^{2 \sigma}\right) .
$$

Applying Hölder inequality and then (2.12) gives

$$
I \leqslant\left.\|r\|_{2}|| u_{2}\right|^{2 \sigma} u_{2}-\left.\left|u_{1}\right|^{2 \sigma} u_{1}\right|_{2} \leqslant\|r\|_{2}\left|\left(\sum_{i=1}^{2}\left|u_{i}\right|^{2 \sigma}\right) r\right|_{2} .
$$


Let $s \in(1,2)$ such that $\frac{4 \sigma}{2-s}=4 \sigma+2$. Applying once more Hölder inequality and then the Sobolev embedding $H^{s}(D) \subset L^{4 \sigma+2}(D)$ gives

$$
I \leqslant\|r\|_{2}|r|_{4 \sigma+2} \sum_{i=1}^{2}\left|u_{i}\right|_{4 \sigma+2}^{2 \sigma} \leqslant\|r\|_{2}\|r\|_{s} \sum_{i=1}^{2}\left|u_{i}\right|_{4 \sigma+2}^{2 \sigma},
$$

which yields by the interpolatory inequality $\|\cdot\|_{s} \leqslant\|\cdot\|_{2}^{s-1}\|\cdot\|^{2-s}$ and then an arithmetic-geometric inequality

$$
I \leqslant\|r\|_{2}^{s}\|r\|^{2-s} \sum_{i}\left|u_{i}\right|_{4 \sigma+2}^{2 \sigma} \leqslant \frac{\varepsilon}{2}\|r\|_{2}^{2}+c\|r\|^{2} \sum_{i}\left|u_{i}\right|_{4 \sigma+2}^{4 \sigma+2} \text {. }
$$

Step 2. We now establish (2.9).

Taking into account (2.8), we see that $r$ satisfies the equation

$$
\frac{\mathrm{d} r}{\mathrm{~d} t}+(\varepsilon+\mathrm{i}) A r=(\eta+\mathrm{i}) Q_{N}\left(\left|u_{2}\right|^{2 \sigma} u_{2}-\left|u_{1}\right|^{2 \sigma} u_{1}\right) .
$$

Taking the scalar product of (2.13) by $-2 A r$, we obtain:

$$
\frac{\mathrm{d}\|r\|^{2}}{\mathrm{~d} t}+2 \varepsilon\|r\|_{2}^{2}=2\left((\eta+\mathrm{i})\left(\left|u_{2}\right|^{2 \sigma} u_{2}-\left|u_{1}\right|^{2 \sigma} u_{1}\right), A r\right) .
$$

Taking into account (2.11), (2.14) gives :

$$
\frac{\mathrm{d}\|r\|^{2}}{\mathrm{~d} t}+\varepsilon\|r\|_{2}^{2} \leqslant c\|r\|^{2} \sum_{i}\left|u_{i}\right|_{4 \sigma+2}^{4 \sigma+2} .
$$

Since $r \in Q_{N} H$, then $\mu_{N+1}\|r\|^{2} \leqslant\|r\|_{2}^{2}$ and it follows from (2.15) that

$$
\frac{\mathrm{d}\|r\|}{\mathrm{d} t}+\varepsilon \mu_{N+1}\|r\|^{2} \leqslant c\|r\|^{2} \sum_{i}\left|u_{i}\right|_{4 \sigma+2}^{4 \sigma+2} .
$$

Applying Gromwall lemma to (2.16), we obtain (2.9).

Proof of Lemma 2.7 in the second case. We first state the following lemma which strengthen Proposition 2.5.

Lemma 2.16. Let $u_{1}$ and $u_{2}$ be two solutions of the CGL system (2.2) associated with $W_{1}$ and $W_{2}$ respectively. If

$$
P_{N} u_{1}(s)=P_{N} u_{2}(s), \quad Q_{N} W_{1}(s)=Q_{N} W_{2}(s), \quad \text { for any } s \in\left(T_{0}, t\right),
$$

where $N$ is a non-negative integer, then

$$
|r(t)|_{L^{2}} \leqslant|r(0)|_{L^{2}} \exp \left(-\frac{\varepsilon \mu_{N+1}}{2} t+c_{1} E_{u_{1}}(t)\right),
$$

where $r=u_{1}-u_{2}$ and where $c_{1}>0$ only depends on $\varepsilon, \eta, \sigma, D$. Moreover, for any $B$, there exists $N_{0}^{\prime \prime}(B, \eta, \varepsilon, D, \sigma)$ such that $N \geqslant N_{0}^{\prime \prime}$ and

$$
E_{u_{1}}(t) \leqslant \rho+B t
$$

imply

$$
|r(t)|_{L^{2}} \leqslant|r(0)|_{L^{2}} \exp \left(-2 t+c_{1} \rho\right)
$$

where $c_{1}$ is the constant of Proposition 2.5.

For the first case, this result is Proposition 1.1.6 of [21]. For the second case the proof is the same.

Sketch of the proof of Lemma 2.16. The proof of Lemma 2.16 is similar to the proof of Proposition 2.5. Indeed it is sufficient to prove

$$
I^{\prime}=-\left((\eta+\lambda \mathrm{i})\left(\left|u_{2}\right|^{2 \sigma} u_{2}-\left|u_{1}\right|^{2 \sigma} u_{1}\right), r\right) \leqslant\left.\left. c|| u_{1}\right|^{2 \sigma}|r|^{2}\right|_{1}
$$


to establish Lemma 2.16. We prove (2.21) as follows. Remarking that

$$
\left|u_{2}\right|^{2 \sigma} u_{2}-\left|u_{1}\right|^{2 \sigma} u_{1}=\left|u_{2}\right|^{2 \sigma} r+u_{1}\left(\left|u_{2}\right|^{2 \sigma}-\left|u_{1}\right|^{2 \sigma}\right),
$$

and

$$
\left|u_{1}\left(\left|u_{2}\right|^{2 \sigma}-\left|u_{1}\right|^{2 \sigma}\right)\right| \leqslant c^{\prime}\left|u_{1}\right|\left(\left|u_{2}\right|^{2 \sigma-1}+\left|u_{1}\right|^{2 \sigma-1}\right)|r|,
$$

we obtain

$$
I^{\prime} \leqslant-\left.\left.\eta|| u_{2}\right|^{2 \sigma}|r|^{2}\right|_{1}+\left.\left.c^{\prime}|| u_{1}\right|^{2 \sigma}|r|^{2}\right|_{1}+\left.\left.c^{\prime}\left|u_{1}\right| u_{2}\right|^{2 \sigma-1} r^{2}\right|_{1} .
$$

Applying arithmetic-geometric inequality to the last term of the previous equality, we obtain for $\sigma \geqslant \frac{1}{2}$

$$
\left.\left.c^{\prime}\left|u_{1}\right| u_{2}\right|^{2 \sigma-1} r^{2}\right|_{1} \leqslant\left.\left.\eta|| u_{2}\right|^{2 \sigma}|r|^{2}\right|_{1}+\left.\left.c^{\prime \prime}|| u_{1}\right|^{2 \sigma}|r|^{2}\right|_{1} .
$$

We infer (2.21) for $\sigma \geqslant \frac{1}{2}$ from the two previous inequalities.

To obtain (2.21) when $\sigma<\frac{1}{2}$, one remark that $D$ is the union of $\left\{x|| u_{1}(x)|\geqslant| u_{2}(x) \mid\right\}$ and $\left\{x|| u_{1}(x)|<| u_{2}(x) \mid\right\}$. Treating the first set is trivial. The treatment done before works for the second set.

Let us set

$$
I=\int_{T_{0}}^{\tau}\left|P_{N}\left(\left|u_{1}(s)\right|^{2 \sigma} u_{1}(s)-\left|u_{2}(s)\right|^{2 \sigma} u_{2}(s)\right)\right|^{2} \mathrm{~d} s .
$$

Applying Lemma 2.16 with the same $N_{0}^{\prime \prime}$, we obtain

$$
|r(t)| \leqslant|r(0)| \exp \left(-2 t+c_{1} \rho\right), \quad \text { for } \tau \geqslant t \geqslant 0 .
$$

Noticing that, since we work in a finite dimensional space, all the norm are equivalent. Hence there exists $K_{N}$ such that

$$
I \leqslant\left. K_{N} \int_{T_{0}}^{\tau}|| u_{1}(s)\right|^{2 \sigma} u_{1}(s)-\left.\left|u_{2}(s)\right|^{2 \sigma} u_{2}(s)\right|_{1} ^{2} \mathrm{~d} s .
$$

It follows from (2.12) and Hölder inequality that

$$
|| u_{1}(s)|-| u_{2}(s)||_{1}^{2} \leqslant c\left|\left(\sum_{i=1}^{2}\left|u_{i}(s)\right|^{2 \sigma}\right)\right| r(s)||_{1}^{2} \leqslant c\left(\sum_{i=1}^{2}\left|u_{i}(s)\right|_{4 \sigma}^{4 \sigma}\right)|r(s)|^{2},
$$

which yields, by applying an arithmetic-geometric inequality,

$$
|| u_{1}(s)|-| u_{2}(s)||_{1}^{2} \leqslant c\left(1+\sum_{i=1}^{2}\left|u_{i}(s)\right|_{4 \sigma+2}^{4 \sigma+2}\right)|r(s)|^{2} .
$$

Combining (2.22), (2.23) and (2.24) and then an integration by parts, we obtain

$$
\begin{aligned}
I & \leqslant K_{N}|r(0)|^{2} \int_{T_{0}}^{\tau} \exp \left(-4 t+c_{1} \rho\right)\left(1+\sum_{i=1}^{2}\left|u_{i}(s)\right|_{4 \sigma+2}^{4 \sigma+2}\right) \mathrm{d} s \\
& \leqslant K_{N}|r(0)|^{2} \int_{T_{0}}^{\tau} \exp \left(-4 t+c_{1} \rho\right)\left(1+\sum_{i=1}^{2} \int_{T_{0}}^{t}\left|u_{i}(s)\right|_{4 \sigma+2}^{4 \sigma+2} \mathrm{~d} s\right) \mathrm{d} t \\
& \leqslant K_{N}|r(0)|^{2} \int_{T_{0}}^{\tau} \exp \left(-4 t+c_{1} \rho\right)\left(1+2 \rho+B t+C\left(1+t^{\alpha}\right)\right) \mathrm{d} t
\end{aligned}
$$




$$
\leqslant K_{N}|r(0)|^{2} \int_{T_{0}}^{\tau} \exp \left(-3 t+2 c_{1} \rho\right) \mathrm{d} t,
$$

which allows us to conclude.

\subsection{An exponential estimate for the growth of solution}

As in the previous subsection, we only give the proof of Propositions 2.8 in the second case.

We set

$$
\begin{aligned}
E_{u}^{\prime}(t)= & \frac{1}{2}\|u(t)\|^{2}+\frac{1}{2 \sigma+2}|u(t)|_{2 \sigma+2}^{2 \sigma+2}+\varepsilon \int_{0}^{t}\|u(s)\|_{2}^{2} \mathrm{~d} s+\eta \int_{0}^{t}\|u(s)\|_{4 \sigma+2}^{4 \sigma+2} \mathrm{~d} s \\
& +(\eta+\varepsilon) \int_{0}^{t} \int_{D}(1+\chi(u \nabla \bar{u}))|u(s, x)|^{2 \sigma}|\nabla u(s, x)|^{2} \mathrm{~d} x \mathrm{~d} s,
\end{aligned}
$$

where $\chi(z)=2 \sigma \Re e\left(\frac{\Re e z}{z}\right)$. Applying Ito's formula to

$$
\mathcal{H}(u)=\frac{1}{2}\|u\|^{2}+\frac{1}{2 \sigma+2}|u|_{2 \sigma+2}^{2 \sigma+2}
$$

we obtain

$$
E_{u}^{\prime}(t)=\mathcal{H}\left(u_{0}\right)+M_{1}(t)+M_{2}(t)+I_{1}(t)+I_{2}(t)
$$

where we have denoted

$$
\begin{array}{ll}
M_{1}(t)=\int_{0}^{t}(-\Delta u(s), b(u(s)), \mathrm{d} W(s)), & \left.M_{2}(t)=\int_{0}^{t}(|u(s)|, b(u(s))) \mathrm{d} W(s)\right), \\
I_{1}(t)=\frac{1}{2} \int_{0}^{t}|b(u(s))|_{\mathcal{L}^{2}\left(L^{2}(D), H^{1}(D)\right)}^{2} \mathrm{~d} s, & I_{2}(t)=\frac{1}{2} \int_{0}^{t} \sum_{i=1}^{2}\left|g_{i}(u(s))\right|_{\mathcal{L}^{2}\left(L^{2}(D)\right)}^{2} \mathrm{~d} s,
\end{array}
$$

where

$$
g_{i}(u)(k)=f_{i}(u)(b(u) h), \quad f_{1}(u)(k)=|u|^{\sigma} \times k, \quad f_{2}(u)(k)=\sqrt{2 \sigma}|u|^{\sigma-1} \Re e(\bar{u} \times k) .
$$

Hölder estimate and Sobolev embedding give

$$
\sum_{i=1}^{2}\left|f_{i}(u)\right|_{\mathcal{L}\left(H^{1}\left(D, L^{2}(D)\right)\right)}^{2} \leqslant c|u|_{4 \sigma+2}^{4 \sigma},
$$

which yields

$$
\sum_{i=1}^{2}\left|g_{i}(u)\right|_{\mathcal{L}^{2}\left(L^{2}(D)\right)}^{2} \leqslant c|u|_{4 \sigma+2}^{4 \sigma} B_{1},
$$

and thus by an arithmetic-geometric inequality

$$
I_{2}(t) \leqslant c B_{1} t+\frac{\eta}{4} \int_{0}^{t}|u|_{4 \sigma+2}^{4 \sigma+2} \mathrm{~d} s .
$$

Notice that

$$
\left\langle M_{1}\right\rangle(t)=\int_{0}^{t}\left|b(u(s))^{*} A u(s)\right|^{2} \mathrm{~d} s,
$$


which gives

$$
\left\langle M_{1}\right\rangle(t) \leqslant B_{0} \int_{0}^{t}\|u(s)\|_{2}^{2} \mathrm{~d} s .
$$

Moreover

$$
\left\langle M_{2}\right\rangle(t)=\left.\left.\int_{0}^{t}\left|b(u(s))^{*}\right| u(s)\right|^{2 \sigma} u(s)\right|^{2} \mathrm{~d} s
$$

Since

$$
\left.\left.\left|b(u(s))^{*}\right| u(s)\right|^{2 \sigma} u(s)\right|^{2} \leqslant\left.\left. B_{0}|| u(s)\right|^{2 \sigma} u(s)\right|^{2} \leqslant c B_{0}|u|_{4 \sigma+2}^{4 \sigma+2},
$$

we obtain

$$
\left\langle M_{2}\right\rangle(t) \leqslant c B_{0} \int_{0}^{t}|u|_{4 \sigma+2}^{4 \sigma+2} \mathrm{~d} s .
$$

Noticing that $\left\langle M_{1}+M_{2}\right\rangle \leqslant 2\left(\left\langle M_{1}\right\rangle+\left\langle M_{2}\right\rangle\right), I_{1}(t) \leqslant B_{1} t$ and $\chi(z) \geqslant 0$ for any $z \in \mathbb{C}$, it follows from (2.25), (2.26), (2.27) and (2.28) that

$$
E_{u}(t)-\mathcal{H}\left(u_{0}\right)-B t \leqslant M(t)-\frac{\gamma_{0}}{2}\langle M\rangle(t),
$$

where $M=M_{1}+M_{2}, B^{\prime}=c\left(B_{0}+B_{1}\right)$ and $\gamma_{0}=\eta \vee \varepsilon /\left(8 B_{0}(1+c)\right)$. Thus

$$
\mathbb{P}\left(\sup _{t \in \mathbb{R}^{+}}\left(E_{u}(t)-B t\right) \geqslant \mathcal{H}\left(u_{0}\right)+\rho^{\prime}\right) \leqslant \mathrm{e}^{-\gamma_{0} \rho^{\prime}} \mathbb{E} \mathrm{e}^{\gamma_{0} M(t)-\gamma_{0}^{2} / 2\langle M\rangle(t)} \leqslant \mathrm{e}^{-\gamma_{0} \rho^{\prime}},
$$

which allows to conclude by setting $\rho^{\prime}=\rho+3 T_{0} / \gamma_{0}$ and $B^{\prime}=B+3 / \gamma_{0}$.

We do not give the proof of Proposition 2.9 because it is easily deduced from the proof of Proposition 2.8. Actually, Ito formulas associated to a solution $u$ are also true if we replace $u$ by $\tilde{u}$ and $b\left(P_{N} u\right) \mathrm{d} W$ by $b\left(P_{N} \tilde{u}\right) \mathrm{d} W+P_{N}\left(|u|^{2 \sigma} u-\right.$ $\left.|\tilde{u}|^{2 \sigma} \tilde{u}\right) \mathrm{d} t$. Hence to establish Proposition 2.9, it is sufficient to bound the additional term by using the equivalence of the norms in finite dimensional spaces and by applying Proposition 2.9 to bound terms containing $u$.

\subsection{The Lyapounov structure}

Now, we prove Lemma 2.10 in the second case. Using the computation of the energy previously done, we obtain that there exist $C_{1}$ such that

$$
\mathrm{d} \mathcal{H}\left(u_{i}(t)\right)+\frac{\varepsilon}{2}\left\|u_{i}(t)\right\|_{2}^{2} \mathrm{~d} t+\frac{\eta}{4 \sigma+2}\left|u_{i}(t)\right|_{4 \sigma+2}^{4 \sigma+2} \mathrm{~d} t \leqslant \mathrm{~d} M+C_{1} \mathrm{~d} t .
$$

Applying Ito formula to $\mathcal{H}\left(u_{i}\right)^{k}$ and controlling $\mathrm{d}\langle M\rangle$ as above by $\left\|u_{i}(t)\right\|_{2}^{2} \mathrm{~d} t$ and $\left|u_{i}(t)\right|_{4 \sigma+2}^{4 \sigma+2} \mathrm{~d} t$, we obtain that there exists $\alpha_{0}$ such that

$$
\mathrm{d} \mathcal{H}\left(u_{i}(t)\right)^{k}+\alpha_{0} k \mathcal{H}\left(u_{i}\right)^{k-1}\left(\left\|u_{i}(t)\right\|_{2}^{2}+\left|u_{i}(t)\right|_{4 \sigma+2}^{4 \sigma+2}\right) \mathrm{d} t \leqslant k \mathcal{H}\left(u_{i}(t)\right)^{k-1} \mathrm{~d} M+C_{k} \mathrm{~d} t .
$$

Taking into account that $\mu_{1}\|\cdot\|^{2} \leqslant\|\cdot\|_{2}^{2}$ and that there exist $\beta>0$ such that $\beta|\cdot|_{2 \sigma+2}^{2 \sigma+2} \leqslant\|\cdot\|_{2}^{2}+|\cdot|_{4 \sigma+2}^{4 \sigma+2}$, we obtain that there exists $\alpha>0$ such that

$$
\mathrm{d} \mathcal{H}\left(u_{i}(t)\right)^{k}+\alpha k \mathcal{H}\left(u_{i}\right)^{k} \mathrm{~d} t \leqslant k \mathcal{H}\left(u_{i}(t)\right)^{k-1} \mathrm{~d} M+C_{k} \mathrm{~d} t .
$$

Now, applying (2.31), we obtain that

$$
\mathcal{H}\left(u_{i}(t)\right)^{k} \leqslant \mathcal{H}\left(u_{0}^{i}\right)^{k} \mathrm{e}^{-\alpha k t}+k \int_{0}^{t} \mathrm{e}^{-\alpha k(t-s)} \mathcal{H}\left(u_{i}(s)\right)^{k-1} \mathrm{~d} M(s)+C_{k} .
$$


Then, applying (2.32) to some stopping time $\tau$, we obtain

$$
\mathbb{E} \mathcal{H}\left(u_{i}(\tau \wedge t)\right)^{k} \leqslant \mathcal{H}\left(u_{0}^{i}\right)^{k} \mathbb{E} \mathrm{e}^{-\tau \wedge t}+C_{k},
$$

Fatou Lemma gives

$$
\mathbb{E} \mathcal{H}\left(u_{i}(\tau)\right)^{k} 1_{\tau<\infty} \leqslant \mathcal{H}\left(u_{0}^{i}\right)^{k} \mathbb{E} \mathrm{e}^{-\tau} 1_{\tau<\infty}+C_{k},
$$

which establishes Lemma 2.10 .

\subsection{Control of $\mathcal{P}_{T} \mathcal{H}$ by $|\cdot|^{2}$ in the second case}

Now, we prove Proposition 2.12. Taking the expectation on (2.29), we obtain that for any $T>t>0$

$$
\mathbb{E} \mathcal{H}(u(T)) \leqslant \mathbb{E} \mathcal{H}(u(t))+B(T-t) .
$$

Integrating over $[0, T]$ gives

$$
\mathbb{E} \mathcal{H}(u(T)) \leqslant \frac{1}{T} \mathbb{E} \int_{0}^{T} \mathcal{H}(u(t)) \mathrm{d} t+B T
$$

Applying Ito formula to $|u|^{2}$ and taking the expectation, we obtain

$$
\mathbb{E}|u(t)|^{2}+2 \varepsilon \int_{0}^{t} \mathbb{E}\|u(s)\|^{2} \mathrm{~d} s+2 \eta \int_{0}^{t} \mathbb{E}|u(s)|_{2 \sigma+2}^{2 \sigma+2} \mathrm{~d} s=\left|u_{0}\right|^{2}+\int_{0}^{t} \mathbb{E}|b(u(s))|_{\mathcal{L}_{2}\left(L^{2}(D)\right)}^{2} \mathrm{~d} s .
$$

Applying $\mathrm{H} 1^{\prime}$, we obtain

$$
\mathbb{E} \int_{0}^{T} \mathcal{H}(u(t)) \mathrm{d} t \leqslant C\left|u_{0}\right|^{2}+A T,
$$

and by 2.35

$$
\mathbb{E} \mathcal{H}(u(T)) \leqslant A+B T+\frac{C}{T}\left|u_{0}\right|^{2}
$$

2.7. $H^{1}$ and $H^{2}$ estimates

We first establish that

$$
\mathbb{E}\|u(T)\|^{2}+\varepsilon \int_{0}^{T} \mathbb{E}\|u(s)\|_{2}^{2} \mathrm{~d} s \leqslant\left\|u_{0}\right\|^{2}+c_{1}\left|u_{0}\right|^{\alpha_{1}}+B_{1}^{\prime} T,
$$

and that

$$
\mathbb{E}\|u(T)\|^{2} \leqslant c\left(1+\frac{1}{T}\left|u_{0}\right|^{2}+\left|u_{0}\right|^{2 k}+T\right) .
$$

In the second part of the proof, we establish that there exists $\gamma_{0}>0$ such that

$$
\mathbb{E}\|u(t)\|_{2}^{2}+\varepsilon \int_{0}^{t} \mathbb{E}\|u(s)\|_{3}^{2} \mathrm{~d} s \leqslant\left\|u_{0}\right\|_{2}^{2}+c\left\|u_{0}\right\|^{\gamma_{0}}+C(t+1) .
$$

We deduce from Hölder inequality that

$$
\mathbb{E}\left(\|u(t)\|_{2}^{2}+\varepsilon \int_{0}^{t}\|u(s)\|_{3}^{2} \mathrm{~d} s\right)^{2 / \gamma_{0}} \leqslant c\left\|u_{0}\right\|_{2}^{2}+C(t+1)
$$


and

$$
\mathbb{E}\|u(T)\|_{2}^{2} \leqslant c\left(1+\frac{1}{T}\left\|u_{0}\right\|^{2}+T\right) .
$$

Hence, combining (2.37), (2.39) and (2.40), we obtain

$$
\mathbb{E}\left(\|u(T+t)\|_{2}^{2}+\varepsilon \int_{T}^{T+t}\|u(s)\|_{3}^{2} \mathrm{~d} s\right)^{2 / \gamma_{0}} \leqslant c \frac{1}{T}\left|u_{0}\right|^{2}+\left|u_{0}\right|^{2 k}+C(T+t+1) .
$$

Applying Hölder inequality allows to conclude.

Proof of (2.36) and (2.37). Note that (2.36) and (2.37) have already been demonstrated in the second case. Then it remains to establish (2.36) in the first case, when $\lambda=-1$.

Remark that Ito's formula applied to $|u|^{2 k}$ gives

$$
\mathbb{E}\left(|u(t)|^{2 k}+\eta k \int_{0}^{t}|u(s)|^{2(k-1)}|u|_{2 \sigma+2}^{2 \sigma+2} \mathrm{~d} s\right) \leqslant\left|u_{0}\right|^{2 k}+B_{k}^{\prime \prime} t .
$$

Taking the scalar product between $(2.2)$ and $2(-\Delta) u$ gives

$$
\mathrm{d}\|u\|^{2}+2 \varepsilon\|u\|_{2}^{2} \mathrm{~d} t \leqslant 2((-\Delta u), b(u) \mathrm{d} W)+2\left(\Delta u,(\eta+\lambda \mathrm{i})|u|^{2 \sigma} u\right) \mathrm{d} t+B_{1} \mathrm{~d} t .
$$

We deduce from Schwartz inequality that

$$
2\left(\Delta u,(\eta+\lambda \mathrm{i})|u|^{2 \sigma} u\right) \leqslant c\|u\|_{2}|u|_{4 \sigma+2}^{2 \sigma+1} .
$$

The Gagliardo-Nirenberg inequality gives

$$
2\left(\Delta u,(\eta+\lambda \mathrm{i})|u|^{2 \sigma} u\right) \leqslant c\|u\|_{2}^{1+\sigma d / 2}|u|^{2 \sigma+1-\sigma d / 2} .
$$

Finally, since $\sigma d<2$, then we can deduce from a arithmetic-geometric inequality that

$$
2\left(\Delta u,(\eta+\lambda \mathrm{i})|u|^{2 \sigma} u\right) \leqslant \varepsilon\|u\|_{2}^{2}+c|u|^{2(4 \sigma+2-\sigma d) /(2-\sigma d)} .
$$

We infer from (2.43) that

$$
\mathrm{d}\|u\|^{2}+\varepsilon\|u\|_{2}^{2} \mathrm{~d} t \leqslant 2((-\Delta u), b(u) \mathrm{d} W)+c|u|^{2(4 \sigma+2-\sigma d) /(2-\sigma d)} \mathrm{d} t+B_{1} \mathrm{~d} t,
$$

and then

$$
\mathbb{E}\|u(t)\|^{2}+\varepsilon \int_{0}^{t} \mathbb{E}\|u(s)\|_{2}^{2} \mathrm{~d} s \leqslant\left\|u_{0}\right\|^{2}+c \int_{0}^{t} \mathbb{E}|u(s)|^{2(4 \sigma+2-\sigma d) /(2-\sigma d)} \mathrm{d} s+B_{1} t .
$$

Applying (2.42), we obtain for a well-chosen $k^{\prime}$

$$
\mathbb{E}\|u(t)\|^{2}+\varepsilon \int_{0}^{t} \mathbb{E}\|u(s)\|_{2}^{2} \mathrm{~d} s \leqslant c\left(\left\|u_{0}\right\|^{2}+\left|u_{0}\right|^{2 k^{\prime}}+T\right) .
$$

Using the same argument as in the last subsection gives (2.37).

Proof of (2.38), (2.39) and (2.40). Taking the scalar product between (2.2) and $2(-\Delta)^{2} u$ gives

$$
\mathrm{d}\|u\|_{2}^{2}+2 \varepsilon\|u\|_{3}^{2} \mathrm{~d} t \leqslant 2\left((-\Delta u)^{2}, b(u) \mathrm{d} W\right)-2\left((-\Delta)^{2} u,(\eta+\lambda \mathrm{i})|u|^{2 \sigma} u\right) \mathrm{d} t+B_{2} \mathrm{~d} t .
$$

We deduce from an integration by part and Schwartz inequality that

$$
-2\left((-\Delta)^{2} u,(\eta+\lambda \mathrm{i})|u|^{2 \sigma} u\right) \leqslant c\|u\|_{3}\left|\nabla\left(u|u|^{2 \sigma}\right)\right| .
$$


Hölder inequality gives

$$
\left|\nabla\left(u|u|^{2 \sigma}\right)\right| \leqslant|\nabla u|_{p}|u|_{2 \sigma q}^{2 \sigma},
$$

where $\frac{1}{p}+\frac{1}{q}=\frac{1}{2}$. We choose $s, p$ and $q$ such that

$$
\frac{1}{p}=\frac{1}{2}-\frac{s}{\mathrm{~d}}, \quad \frac{1}{2 \sigma q}=0 \vee\left(\frac{1}{2}-\frac{1}{d}\right)
$$

Since $\sigma \leqslant \frac{2}{\mathrm{~d}-2}$, then $s \in[0,2)$. Hence the Sobolev embeddings $H^{s}(D) \rightarrow L^{p}(D)$ and $H^{1}(D) \rightarrow L^{2 \sigma q}(D)$ imply

$$
\left.|\nabla u| u\right|^{2 \sigma} \mid \leqslant\|u\|_{1+s}\|u\|^{2 \sigma},
$$

Then, we deduce from (2.46), an interpolatory inequality that

$$
-2\left((-\Delta)^{2} u,(\eta+\lambda \mathrm{i})|u|^{2 \sigma} u\right) \leqslant c\|u\|_{3}^{1+s / 2}\|u\|^{2 \sigma+1-s / 2} .
$$

An arithmetic-geometric inequality gives

$$
-2\left((-\Delta)^{2} u,(\eta+\lambda \mathrm{i})|u|^{2 \sigma} u\right) \leqslant \varepsilon\|u\|_{3}^{2}+c\|u\|^{\beta},
$$

with $\beta>0$. We infer from (2.45) and (2.47) that

$$
\mathrm{d}\|u\|_{2}^{2}+\varepsilon\|u\|_{3}^{2} \mathrm{~d} t \leqslant 2\left((-\Delta u)^{2}, b(u) \mathrm{d} W\right)+c\|u\|^{\beta} \mathrm{d} t+B_{2} \mathrm{~d} t .
$$

Hence, we deduce (2.38) from (2.48). Then, applying Hölder inequality, we obtain (2.39). Using the same argument as in the last subsection gives (2.40).

\section{The coupling of CGL}

Recall that, as in the last section, we consider the two cases developed in Subsection 2.1 and use the properties stated in Subsection 2.2. In this section, we make an other assumption

H4 $N \geqslant N_{0}$, where $N_{0}$ has been defined after Corollary 2.6 and Lemma 2.7.

In this section, we apply Theorem 1.8. Then we obtain there exists a unique invariant probability measure on $H$ and that there exists $c>0$ and $\alpha>0$

$$
\mathbb{P}\left(\left|u_{1}(t)-u_{2}(t)\right|_{H}>c \mathrm{e}^{-\alpha t}\right) \leqslant c \mathrm{e}^{-\alpha t}\left(1+\mathcal{H}\left(u_{0}^{1}\right)+\mathcal{H}\left(u_{0}^{2}\right)\right) .
$$

Recalling Corollary 2.14, we obtain for any $\delta>0$,

$$
\mathbb{P}\left(\left\|u_{i}(t)\right\|_{H^{2}(D)} \geqslant \mathrm{e}^{\delta t}\right) \leqslant C_{\delta} \mathrm{e}^{-\frac{\delta}{\gamma} t}\left(\left|u_{0}^{i}\right|_{L^{2}(D)}^{2}+1\right) .
$$

Combining (3.1), (3.2) and using an interpolatory inequality between $L^{2}(D)$ and $H^{2}(D)$, we obtain that for any $s \in[0,2)$, there exists $\alpha_{s}>0$ and $C_{s}>0$ such that

$$
\mathbb{P}\left(\left\|u_{1}(t)-u_{2}(t)\right\|_{H^{s}(D)}>c \mathrm{e}^{-\alpha_{s} t}\right) \leqslant C_{s} \mathrm{e}^{-\alpha_{s} t}\left(1+\sum_{i=1}^{2}\left(\left|u_{0}^{i}\right|_{L^{2}(D)}^{2}+\mathcal{H}\left(u_{0}^{i}\right)\right)\right),
$$

which implies

$$
\left\|\mathcal{P}_{t}^{*} \mu-v\right\|_{\operatorname{Lip}_{b}\left(H^{s}(D)\right)}^{*} \leqslant C_{s} \mathrm{e}^{-\alpha_{s} t}\left(1+\int_{H}\left(|u|_{L^{2}(D)}^{2}+\mathcal{H}(u)\right) \mathrm{d} \mu(u)\right) .
$$

Now it remains to conclude the second case, we consider $(u, W)$ a weak solution and we apply Proposition 2.12

$$
\mathbb{E}\left(\mathcal{H}(u(T))+|u(T)|_{L^{2}(D)}^{2}\right) \leqslant \frac{1}{T} \mathbb{E}\left|u_{0}\right|_{L^{2}(D)}^{2}+C(1+T),
$$


which implies for all cases

$$
\left|\mathbb{E} f(u(t))-\int_{H} f(u) \mathrm{d} v(u)\right| \leqslant c_{s}|f|_{\operatorname{Lip}_{b}\left(H^{s}(D)\right)} \mathrm{e}^{-\alpha_{s} t}\left(1+\mathbb{E}\left|u_{0}\right|_{L^{2}(D)}^{2}\right),
$$

for any $s<2$, for any $f \in \operatorname{Lip}_{b}\left(H^{s}(D)\right)$.

It follows from this discussion that it suffices to prove that Theorem 1.8 can be applied and that (3.1) holds. Then Theorem 2.1 is proved.

\subsection{Preliminaries}

We set $|\cdot|=|\cdot|_{H}$ and

$$
X=P_{N} u, \quad Y=Q_{N} u, \quad \beta=P_{N} W, \quad \eta=Q_{N} W, \quad \sigma_{l}=P_{N} b P_{N}, \quad \sigma_{h}=Q_{N} b Q_{N},
$$

and

$$
\begin{aligned}
& f(X, Y)=(\eta+\lambda \mathrm{i}) P_{N}\left(|X+Y|^{2 \sigma}(X+Y)\right), \\
& g(X, Y)=(\eta+\lambda \mathrm{i}) Q_{N}\left(|X+Y|^{2 \sigma}(X+Y)\right) .
\end{aligned}
$$

Now, taking into account $\mathrm{H} 2$ and $\mathrm{H} 3$, the system has the form

$$
\left\{\begin{array}{l}
\mathrm{d} X+(\varepsilon+\mathrm{i}) A X \mathrm{~d} t+f(X, Y) \mathrm{d} t=\sigma_{l}(X) \mathrm{d} \beta, \\
\mathrm{d} Y+(\varepsilon+\mathrm{i}) A Y \mathrm{~d} t+g(X, Y) \mathrm{d} t=\sigma_{h}(X) \mathrm{d} \eta \\
X(0)=x_{0}, \quad Y(0)=y_{0}
\end{array}\right.
$$

Recall that $\mathrm{H} 3$ states that

There exists $\sigma_{0}>0$ such that, $\left|\left(\sigma_{l}(x)\right)^{-1}\right| \leqslant \frac{1}{\sigma_{0}}, \quad$ for any $x \in P_{N} H$.

Now we can define $l_{0}$

$$
l_{0}(k)=\min \left\{l \in\{0, \ldots, k\} \mid P_{l, k}\right\},
$$

where $\min \phi=\infty$ and

$$
\left(P_{l, k}\right)\left\{\begin{array}{l}
X_{1}(t)=X_{2}(t), \quad \eta_{1}(t)=\eta_{2}(t), \quad \forall t \in[l T, k T], \\
\mathcal{H}_{l} \leqslant d_{0}, \quad i=1,2, \\
E_{u_{i}}(t+l T, l T) \leqslant \aleph 1_{t<T}+B t+1_{i=2} 1_{t \leqslant T} C_{N}\left(1+t^{\alpha}\right), \quad \forall t \in[0,(k-l) T],
\end{array}\right.
$$

where $B, \alpha, C_{N}$ are defined in Propositions 2.8 and 2.9, where $\aleph$ will be chosen later and where

$$
\mathcal{H}_{k}=\mathcal{H}\left(u_{1}(k T)\right)+\mathcal{H}\left(u_{2}(k T)\right) .
$$

Notice that (1.45) is obvious. Corollary 2.6 and $\mathrm{H} 4$ gives

$$
l_{0}(k)=l \quad \text { implies } \quad\left|u_{1}(t)-u_{2}(t)\right| \leqslant C\left(d_{0}\right) \mathrm{e}^{-(t-l T)}, \quad \text { for any } t \in[l T, k T],
$$

and we have establish (1.46). Lemma 2.10 implies the Lyapounov structure (1.49).

From now on we say that $\left(X_{1}, X_{2}\right)$ are coupled at $k T$ if $l_{0}(k) \leqslant k$, in other words if $l_{0}(k) \neq \infty$. Now it remains to build a coupling such that (3.6) and (3.7) holds, where

$$
\left\{\begin{array}{l}
\forall d_{0}, \exists p_{0}\left(d_{0}\right)>0,\left(p_{i}\right)_{i \in \mathbb{N}^{*}}, T_{0}\left(d_{0}\right)>0 \text { such that for any } l \leqslant k, \\
\mathbb{P}\left(l_{0}(k+1)=l \mid l_{0}(k)=l\right) \geqslant p_{k-l}, \quad \text { for any } T \geqslant T_{0}\left(d_{0}\right), \\
1-p_{i} \leqslant \mathrm{e}^{-i T}, \quad i \in \mathbb{N}^{*}
\end{array}\right.
$$

and, for any $\left(R_{0}, d_{0}\right)$ sufficiently large,

$$
\left\{\begin{array}{l}
\exists T^{*}\left(R_{0}\right)>0 \text { and } p_{-1}>0 \text { such that for any } T \geqslant T^{*}\left(R_{0}\right) \\
\mathbb{P}\left(l_{0}(k+1)=k+1 \mid l_{0}(k)=\infty, \mathcal{H}_{k} \leqslant R_{0}\right) \geqslant p_{-1}
\end{array}\right.
$$

These properties imply (1.47) and (1.48) and Theorem 1.8 can be applied. 
As in the example of Section 1.2, we remark that by induction, it suffices to construct a probability space $\left(\Omega_{0}, \mathcal{F}_{0}, \mathbb{P}_{0}\right)$ and two measurable couples of functions $\left(\omega_{0}, u_{0}^{1}, u_{0}^{2}\right) \rightarrow\left(V_{i}\left(\cdot, u_{0}^{1}, u_{0}^{2}\right)\right)_{i=1,2}$ and $\left(V_{i}^{\prime}\left(\cdot, u_{0}^{1}, u_{0}^{2}\right)\right)_{i=1,2}$ and such that, for any $\left(u_{0}^{1}, u_{0}^{2}\right),\left(V_{i}\left(\cdot, u_{0}^{1}, u_{0}^{2}\right)\right)_{i=1,2}$ and $\left(V_{i}^{\prime}\left(\cdot, u_{0}^{1}, u_{0}^{2}\right)\right)_{i=1,2}$ are two couplings of $\left(\mathcal{D}\left(u\left(\cdot, u_{0}^{i}\right), W\right)\right)_{i=1,2}$ on $[0, T]$. Indeed, we first set

$$
u_{i}(0)=u_{0}^{i}, \quad W_{i}(0)=0, \quad i=1,2 .
$$

Assuming that we have built $\left(u_{i}, W_{i}\right)_{i=1,2}$ on $[0, k T]$, then we take $\left(V_{i}\right)_{i}$ and $\left(V_{i}^{\prime}\right)_{i}$ as above independent of $\left(u_{i}, W_{i}\right)_{i=1,2}$ on $[0, k T]$ and set

$$
\left(u_{i}(k T+t), W_{i}(k T+t)\right)= \begin{cases}V_{i}\left(t, u_{1}(k T), u_{2}(k T)\right) & \text { if } l_{0}(k) \leqslant k, \\ V_{i}^{\prime}\left(t, u_{1}(k T), u_{2}(k T)\right) & \text { if } l_{0}(k)=\infty,\end{cases}
$$

for any $t \in[0, T]$.

\subsection{Proof of (3.6)}

The essential difference between this proof and the proof of (1.18) in the example in Section 1.2 is that a cut-off is used to control the energy.

To build $\left(V_{i}\left(\cdot, u_{0}^{1}, u_{0}^{2}\right)\right)_{i=1,2}$, we apply Proposition 1.7 to

$$
\begin{aligned}
& E=C((0, T) ; H) \times C\left((0, T) ; H^{-d / 2-1}(D)\right), \\
& F=C\left((0, T) ; P_{N} H\right) \times C\left((0, T) ; Q_{N} H^{-d / 2-1}(D)\right), \\
& f_{0}(u, W)=(X, \eta), \\
& \mu_{i}=\mathcal{D}\left(u\left(\cdot, u_{0}^{i}\right), W\right), \quad \text { on }[0, T] .
\end{aligned}
$$

Remark that if we set $v_{i}=f_{0}^{*} \mu_{i}$, we obtain

$$
v_{i}=\mathcal{D}\left(X\left(\cdot, u_{0}^{i}\right), \eta\right), \quad \text { on }[0, T] .
$$

We set

$$
\left(Z_{i}, \xi_{i}\right)=f_{0}\left(V_{i}\right), \quad i=1,2 .
$$

Then $\left(V_{i}\left(\cdot, u_{0}^{1}, u_{0}^{2}\right)\right)_{i=1,2}$ is a coupling of $\left(\mu_{1}, \mu_{2}\right)$ such that $\left(\left(Z_{i}, \xi_{i}\right)\left(\cdot, u_{0}^{1}, u_{0}^{2}\right)\right)_{i=1,2}$ is a maximal coupling of $\left(v_{1}, v_{2}\right)$.

We first use a Girsanov formula to estimate $I_{p}$, where

$$
\begin{aligned}
& I_{p}=\int_{A_{k, l}}\left(\frac{\mathrm{d} \nu_{2}}{\mathrm{~d} \nu_{1}}\right)^{p+1} \mathrm{~d} v_{2}, \\
& A_{k, l}=\left\{(Z, \xi) \mid \tau_{k, l}=T\right\}, \\
& \tau_{k, l}=\inf \left\{t \in[0, T] \mid E_{\hat{u}_{i}}(t+k T, l T)>\aleph 1_{k=l}+B(t+(k-l) T)+1_{i=2} 1_{k=l} C_{N}\left(1+t^{\alpha}\right), i \in\{1,2\}\right\},
\end{aligned}
$$

where

$$
\hat{u}_{i}=u_{i} \quad \text { on }[0, k T], \quad \hat{u}_{i}(k T+\cdot)=Z+\Phi\left(Z, \xi, u_{0}^{i}\right) \quad \text { on }[0, T] .
$$

Then, using Lemma 1.2, we establish (3.6).

We consider a couple of $\left(u_{i}, W_{i}\right)_{i=1,2}$, two solutions of (3.3) on $[0, k T]$ and a trajectory of $\left(u_{i}, W_{i}\right)_{i=1,2}$ such that $l_{0}(k)=l$. We set

$$
x=X_{1}(k T)=X_{2}(k T), \quad y_{i}=Y_{i}(k T), \quad i=1,2 .
$$

Let $W=(\beta, \xi)$ a cylindrical Wiener process defined on a probability space $(\Omega, \mathcal{F}, \mathbb{P})$. We denote by $Z$ the unique solution of the truncated equation

$$
\left\{\begin{array}{l}
\mathrm{d} Z+(\varepsilon+\mathrm{i}) A Z \mathrm{~d} t+1_{t \leqslant \tau_{k, l}} f\left(Z, \Phi\left(Z, \xi,\left(x, y_{1}\right)\right)\right) \mathrm{d} t=\sigma_{l}(Z) \mathrm{d} \beta \\
Z(0)=x
\end{array}\right.
$$


We denote by $\lambda_{1}$ the distribution of $(Z, \xi)$ under the probability $\mathbb{P}$.

We set $\tilde{\beta}(t)=\beta(t)+\int_{0}^{t} d(s) \mathrm{d} t$ where

$$
\mathrm{d}(t)=1_{t \leqslant \tau_{k, l}}\left(\sigma_{l}(Z(t))\right)^{-1}\left(f\left(Z(t), \Phi\left(Z, \xi,\left(x, y_{2}\right)\right)(t)\right)-f\left(Z(t), \Phi\left(Z, \xi,\left(x, y_{1}\right)\right)(t)\right)\right) .
$$

Then $Z$ is a solution of

$$
\left\{\begin{array}{l}
\mathrm{d} Z+(\varepsilon+\mathrm{i}) A Z \mathrm{~d} t+1_{t \leqslant \tau_{k, l}} f\left(Z, \Phi\left(Z, \xi,\left(x, y_{2}\right)\right)\right) \mathrm{d} t=\sigma_{l}(Z) \mathrm{d} \tilde{\beta} \\
Z(0)=x
\end{array}\right.
$$

The drift estimate in Lemma 2.7 ensures that

$$
\int_{0}^{T}|d(t)|^{2} \mathrm{~d} t \leqslant c d_{0} \sigma_{0}^{-2} \exp \left(-3(k-l) T+c \aleph 1_{k=l}\right) .
$$

Hence the Novikov condition is satisfied and the Girsanov formula can be applied. Then we set

$$
\mathrm{d} \widetilde{\mathbb{P}}=\exp \left(\int_{0}^{T} d(s) \mathrm{d} W(s)-\frac{1}{2} \int_{0}^{T}|d(s)|^{2} \mathrm{~d} t\right) \mathrm{d} \mathbb{P} .
$$

We deduce from the Girsanov formula that $\widetilde{\mathbb{P}}$ is a probability under which $(\tilde{\beta}, \xi)$ is a cylindrical Wiener process and we denote by $\lambda_{2}$ the law of $(Z, \xi)$ under $\widetilde{\mathbb{P}}$. Moreover, remarking that

$$
\lambda_{i}\left(A_{k, l} \cap \cdot\right)=v_{i}\left(A_{k, l} \cap \cdot\right), \quad i=1,2,
$$

we obtain

$$
I_{p} \leqslant I_{p}^{\prime} \leqslant \mathbb{E} \exp \left(c_{p} \int_{0}^{T}|d(s)|^{2} \mathrm{~d} t\right)
$$

where

$$
I_{p}^{\prime}=\int_{F}\left(\frac{\mathrm{d} \lambda_{2}}{\mathrm{~d} \lambda_{1}}\right)^{p+1} \mathrm{~d} \lambda_{2}
$$

Then it follows from (3.11) that

$$
I_{p} \leqslant I_{p}^{\prime} \leqslant \exp \left(c_{p} \sigma_{0}^{-2} d_{0} \mathrm{e}^{-3(k-l) T+c \kappa 1_{k=l}}\right) .
$$

Notice that

$$
\left\|\lambda_{1}-\lambda_{2}\right\|_{\mathrm{var}}=\int_{F}\left|\frac{\mathrm{d} \lambda_{2}}{\mathrm{~d} \lambda_{1}}-1\right| \mathrm{d} \lambda_{2} \leqslant \sqrt{\int\left(\frac{\mathrm{d} \lambda_{2}}{\mathrm{~d} \lambda_{1}}\right)^{2} \mathrm{~d} \lambda_{2}-1} .
$$

We infer from (3.14) that, for $T \geqslant T_{3}\left(d_{0}\right)=2 \ln \left(c_{p} \sigma_{0}^{-2} d_{0}\right)$,

$$
\left\|\lambda_{1}-\lambda_{2}\right\|_{\text {var }} \leqslant \frac{1}{2} \mathrm{e}^{-2(k-l) T}
$$

Using (3.12), we obtain for $k>l$

$$
\left\|v_{1}-v_{2}\right\|_{\mathrm{var}} \leqslant\left\|\lambda_{1}-\lambda_{2}\right\|_{\mathrm{var}}+\sum_{i=1}^{2} v_{i}\left(A_{k, l}^{i}\right) \leqslant \frac{1}{2} \mathrm{e}^{-2(k-l) T}+\sum_{i=1}^{2} v_{i}\left(A_{k, l}^{i}\right),
$$

where

$$
A_{k, l}^{i}=\left\{(Z, \xi) \mid E_{Z+\phi\left(Z, \xi, u_{0}^{i}\right)}(t, l T) \leqslant B(t+(k-l) T) \text { for any } t \in[0, T]\right\} .
$$


Applying Lemma 1.2 to the maximal coupling $\left(Z_{1}, Z_{2}\right)_{i=1,2}$ of $\left(v_{1}, v_{2}\right)$ gives for $k>l$

$$
\mathbb{P}\left(\left(Z_{1}, \xi_{1}\right) \neq\left(Z_{2}, \xi_{2}\right)\right) \leqslant\left\|v_{1}-v_{2}\right\|_{\text {var }} \leqslant \frac{1}{2} \mathrm{e}^{-2(k-l) T}+\sum_{i=1}^{2} v_{i}\left(A_{k, l}^{i}\right) .
$$

Using (3.8) and (3.15), we obtain that on $l_{0}(k)=l$

$$
\mathbb{P}\left(\left(X_{1}, \eta_{1}\right) \neq\left(X_{2}, \eta_{2}\right) \text { on }[k T,(k+1) T] \mid \mathcal{F}_{k T}\right) \leqslant \frac{1}{2} \mathrm{e}^{-2(k-l) T}+2 \mathbb{P}\left(B_{l, k} \mid \mathcal{F}_{k T}\right),
$$

where

$$
B_{l, k}=\left\{E_{u_{i}}(t, l T) \leqslant B(t-l T), \text { for any } t \in[k T,(k+1) T], i=1,2\right\} .
$$

Noticing that for $k>l$

$$
\left\{l_{0}(k+1)=l\right\}=\left\{l_{0}(k)=l\right\} \cap\left\{\left(X_{1}, \eta_{1}\right)=\left(X_{2}, \eta_{2}\right) \text { on }[k T,(k+1) T]\right\} \cap B_{l, k},
$$

and integrating over $l_{0}(k)=l$ gives for $T \geqslant T_{1}\left(d_{0}\right)$ and for $k>l$

$$
\mathbb{P}\left(l_{0}(k+1) \neq l \mid l_{0}(k)=l\right) \leqslant \frac{1}{2} \mathrm{e}^{-2(k-l) T}+3 \mathbb{P}\left(B_{l, k} \mid l_{0}(k)=l\right),
$$

and then

$$
\mathbb{P}\left(l_{0}(k+1) \neq l, l_{0}(k)=l \mid l_{0}(l)=l\right) \leqslant \frac{1}{2} \mathrm{e}^{-2(k-l) T}+3 \mathbb{P}\left(B_{l, k} \mid l_{0}(l)=l\right) .
$$

The exponential estimate for growth of the solution (Proposition 2.8) gives that for $T$ sufficiently high

$$
\mathbb{P}\left(l_{0}(k+1) \neq l, l_{0}(k)=l \mid l_{0}(l)=l\right) \leqslant \exp (-2(k-l) T) .
$$

Now, it remains to consider the case $k=l$, we apply Lemmas 1.2 and 1.3 to $\left(Z_{i}, \xi_{i}\right)_{i=1,2}$ which gives

$$
\mathbb{P}\left(\left(Z_{1}, \xi_{1}\right)=\left(Z_{2}, \xi_{2}\right), A_{l, l}^{2}\right) \geqslant\left(v_{1} \wedge v_{2}\right)\left(A_{l, l}\right) \geqslant\left(1-\frac{1}{p}\right)\left(p I_{p}\right)^{-1 /(p-1)} v_{1}\left(A_{l, l}\right)^{p /(p-1)}
$$

Choosing $\aleph$ sufficiently high and applying the exponential for growth of the solution (Propositions 2.8 and 2.9), we obtain

$$
v_{1}\left(A_{l, l}\right) \geqslant \frac{1}{2},
$$

and then applying (3.13) and fixing $p>1$,

$$
\mathbb{P}\left(\left(Z_{1}, \xi_{1}\right)=\left(Z_{2}, \xi_{2}\right), A_{l, l}\right) \geqslant p_{0}\left(d_{0}\right)>0 .
$$

That gives

$$
\mathbb{P}\left(l_{0}(l+1)=l \mid l_{0}(l)=l\right) \geqslant p_{0}\left(d_{0}\right)>0 .
$$

Since

$$
\mathbb{P}\left(l_{0}(k) \neq l \mid l_{0}(l)=l\right) \leqslant \sum_{n=l}^{k-1} \mathbb{P}\left(l_{0}(n+1) \neq l, l_{0}(n)=l \mid l_{0}(l)=l\right),
$$

then, by applying (3.16) and (3.17), we obtain

$$
\mathbb{P}\left(l_{0}(k) \neq l \mid l_{0}(l)=l\right) \leqslant 1-p_{0}+\sum_{n=1}^{\infty} \exp (-2 n T) \leqslant 1-p_{0}+\frac{\exp (-2 T)}{1-\exp (-2 T)},
$$

which implies that for $T \geqslant T_{0}\left(d_{0}\right)$

$$
\mathbb{P}\left(l_{0}(k)=l \mid l_{0}(l)=l\right) \geqslant \frac{p_{0}}{2} .
$$

Combining (3.16), (3.17) and (3.18), we establish (3.6) for $T$ sufficiently high. 


\subsection{Proof of (3.7)}

As in the example of Section 1.2, The Lyapounov structure gives that it is sufficient to find $d_{0}>0, \tilde{p}>0, R_{1}>4 K_{1}$ and a coupling $\left(V_{i}\left(\cdot, u_{0}^{1}, u_{0}^{2}\right)\right)_{i=1,2}$ of $\left(\mu_{1}, \mu_{2}\right)$, where

$$
\mu_{i}=\mathcal{D}\left(u\left(\cdot, u_{0}^{i}\right), W\right), \quad \text { on }[0,1], i=1,2,
$$

and such that

$$
\mathbb{P}\left(Z_{1}\left(1, u_{0}^{1}, u_{0}^{2}\right)=Z_{2}\left(1, u_{0}^{1}, u_{0}^{2}\right), \sum_{i=1}^{2} \mathcal{H}\left(u_{i}\left(1, u_{0}^{1}, u_{0}^{2}\right)\right) \leqslant d_{0}\right) \geqslant \tilde{p},
$$

where

$$
V_{i}\left(\cdot, u_{0}^{1}, u_{0}^{2}\right)=\left(u_{i}\left(\cdot, u_{0}^{1}, u_{0}^{2}\right), W_{i}\left(\cdot, u_{0}^{1}, u_{0}^{2}\right)\right), u_{i}\left(\cdot, u_{0}^{1}, u_{0}^{2}\right)=\left(\begin{array}{c}
Z_{i} \\
G_{i}
\end{array}\right), \quad i=1,2 .
$$

Now we fix $R_{1}>4 K_{1}$ and consider a cimetery value $\Delta$ (some people prefer calling it a heaven value). To build $\left(V_{i}\left(\cdot, u_{0}^{1}, u_{0}^{2}\right)\right)_{i=1,2}$, we apply Proposition 1.7 to

$$
\begin{aligned}
& E=C((0,1) ; H) \times C\left((0,1) ; H^{-d / 2-1}(D)\right), \\
& F=\left(P_{N} H \times C\left((0,1) ; Q_{N} H^{-d / 2-1}(D)\right)\right) \cup\{\Delta\}, \\
& f_{0}(u, W)=X(1) 1_{A}(X, \eta)+\Delta 1_{A^{c}}(X, \eta),
\end{aligned}
$$

and to $\mu_{i}$ where

$$
\begin{aligned}
& A=\{(X, \eta) \mid \tau=1\}, \\
& \tau=\inf \left\{t \in[0,1] \mid E_{X+\Phi\left(X, \eta, u_{0}^{i}\right)}(t)>\aleph+B t+1_{i=2} C_{N}\left(1+t^{\alpha}\right), i \in\{1,2\}\right\} .
\end{aligned}
$$

We set $v_{i}=f_{0}^{*} \mu_{i}$. Then $\left(V_{i}\left(\cdot, u_{0}^{1}, u_{0}^{2}\right)\right)_{i=1,2}$ is a coupling of $\left(\mu_{1}, \mu_{2}\right)$ such that $\left(Z_{i}\left(1, u_{0}^{1}, u_{0}^{2}\right)\right)_{i=1,2}$ is a maximal coupling of $\left(v_{1}, v_{2}\right)$.

Now, we define

$$
f_{1}(u, W)=(X, \eta) \quad \text { and } \quad f_{2}(X, \eta)=X(1) 1_{A}(X, \eta)+\Delta 1_{A^{c}}(X, \eta),
$$

and we set $\theta_{i}=f_{1}^{*} \mu_{i}$ for $i=1,2$. Now we consider $\left(\hat{\theta}_{1}, \hat{\theta}_{2}\right)$ such that $\theta_{i}(A \cap \cdot)$ is equivalent to $\hat{\theta}_{i}(A \cap \cdot)$ for $i=1,2$ and such that $\left(\hat{v}_{1}, \hat{v}_{2}\right)=\left(f_{2}^{*} \hat{\theta}_{1}, f_{2}^{*} \hat{\theta}_{2}\right)$ are two equivalent measures. Then by applying two Schwartz inequalities, we obtain that

$$
I_{p} \leqslant\left(J_{2 p+2}^{1}\right)^{1 / 2}\left(J_{4 p}^{2}\right)^{1 / 4}\left(\hat{I}_{4 p+2}\right)^{1 / 4},
$$

where

$$
\begin{array}{ll}
I_{p}=\int_{B^{\prime}}\left(\frac{\mathrm{d} \nu_{1}}{\mathrm{~d} \nu_{2}}\right)^{p+1} \mathrm{~d} \nu_{2}, & J_{p}^{1}=\int_{A}\left(\frac{\mathrm{d} \theta_{1}}{\mathrm{~d} \hat{\theta}_{1}}\right)^{p} \mathrm{~d} \hat{\theta}_{1}, \\
\hat{I}_{p}=\int_{B^{\prime}}\left(\frac{\mathrm{d} \hat{v}_{1}}{\mathrm{~d} \hat{v}_{2}}\right)^{p} \mathrm{~d} \hat{v}_{2}, & J_{p}^{2}=\int_{A}\left(\frac{\mathrm{d} \hat{\theta}_{2}}{\mathrm{~d} \theta_{2}}\right)^{p} \mathrm{~d} \hat{\theta}_{2},
\end{array}
$$

Let us consider $\bar{Z}_{i}$ the unique solution of

$$
\left\{\begin{array}{l}
\mathrm{d} \bar{Z}_{i}+(\varepsilon+\mathrm{i}) A \bar{Z}_{i} \mathrm{~d} t+1_{t \leqslant \tau} f\left(\bar{Z}_{i}, \Phi\left(\bar{Z}_{i}(\cdot), \xi(\cdot), u_{0}^{i}\right)\right) \mathrm{d} t=\sigma_{l}\left(\bar{Z}_{i}\right) \mathrm{d} \beta_{i}, \\
\bar{Z}_{i}(0)=x_{0}^{i} .
\end{array}\right.
$$

Taking into account (3.9), we denote by $\lambda_{i}$ the distribution of $\left(\bar{Z}_{i}, \xi_{i}\right)$ under the probability $\mathbb{P}$ and we obtain

$$
\theta_{i}(A \cap \cdot)=\lambda_{i}(A \cap \cdot) .
$$


We set $\tilde{\beta}_{i}(t)=\beta_{i}(t)+\int_{0}^{t} d_{i}(s) \mathrm{d} t$ where

$$
\mathrm{d}_{i}(t)=-1_{t \leqslant \tau}\left(\sigma_{l}\left(\bar{Z}_{i}(t)\right)\right)^{-1} f\left(\bar{Z}_{i}(t), \Phi\left(\bar{Z}_{i}(\cdot), \xi(\cdot), u_{0}^{i}\right)(t)\right) .
$$

Then $\bar{Z}_{i}$ is a solution of

$$
\left\{\begin{array}{l}
\mathrm{d} \bar{Z}_{i}+(\varepsilon+\mathrm{i}) A \bar{Z}_{i} \mathrm{~d} t=\sigma_{l}\left(\bar{Z}_{i}\right) \mathrm{d} \tilde{\beta}_{i}, \\
\bar{Z}_{i}(0)=x_{0}^{i} .
\end{array}\right.
$$

Since the energy is bounded and $\sigma_{l}$ is bounded below, then $d$ is uniformly bounded. Hence, the Novikov condition is satisfied and the Girsanov formula can be applied. Then we set

$$
\mathrm{d} \widetilde{\mathbb{P}}_{i}=\exp \left(\int_{0}^{T} d_{i}(s) \mathrm{d} W(s)-\frac{1}{2} \int_{0}^{T}|d(s)|^{2} \mathrm{~d} t\right) \mathrm{d} \mathbb{P} .
$$

We deduce from the Girsanov formula that $\widetilde{\mathbb{P}}$ is a probability under which $(\tilde{\beta}, \xi)$ is a cylindrical Wiener process. We denote by $\hat{\theta}_{i}$ the law of $\left(\bar{Z}_{i}, \xi_{i}\right)$ under $\widetilde{\mathbb{P}}_{i}$. Moreover using (3.22), we obtain

$$
J_{p}^{1} \vee J_{p}^{2} \leqslant \mathbb{E} \exp \left(c_{p} \int_{0}^{T}|d(s)|^{2} \mathrm{~d} t\right) \leqslant C\left(p, \aleph, R_{1}\right) .
$$

We set $\hat{v}_{i}=f_{2}^{*} \hat{\theta}_{i}$ for $i=1,2$. It is classical that $\hat{v}_{i}$ has a density $q\left(x_{0}^{i}, z\right)$ with respect to Lebesgue measure $\mathrm{d} z$, that $q$ is continuous for the couple $\left(x_{0}^{i}, z\right)$, where $x_{0}^{i}$ is the initial value and where $z$ is the target value and that $q>0$. Then, we can bound $q$ and $q^{-1}$ uniformly on $\mathcal{H}\left(x_{0}^{i}\right) \leqslant R_{1}$ and on $z \in B^{\prime}=\{\mathcal{H}(z) \leqslant C\}$ provided $C=C(\aleph)$. It allows us to bound $\hat{I}_{p}$ and then $I_{p}$. Actually, $d_{1} \geqslant d_{1}(\aleph)$ implies

$$
A \subset B
$$

where

$$
B=\left\{(Z, \xi) \mid \mathcal{H}\left(Z(1)+\phi\left(Z, \xi, u_{0}^{i}\right)(1)\right) \leqslant d_{1}, i=1,2\right\} .
$$

Hence it follows that for $d_{1} \geqslant d_{1}(\aleph)$

$$
I_{p} \leqslant C^{\prime}\left(p, \aleph, R_{1}\right)<\infty .
$$

Now we apply Lemmas 1.3 and 1.2:

$$
\mathbb{P}\left(Z_{1}(1)=Z_{2}(1),\left(A \cap B^{\prime}\right)^{2}\right) \geqslant\left(1-\frac{1}{p}\right)\left(p I_{p}\right)^{-1 /(p-1)} v_{1}\left(B^{\prime}\right)^{p /(p-1)} .
$$

We deduce from Propositions 2.8 and 2.9 and from $C(\aleph) \rightarrow \infty$ when $\aleph \rightarrow \infty$ that $\aleph$ sufficiently high gives

$$
v_{1}\left(B^{\prime}\right) \geqslant \frac{1}{2}
$$

Combining (3.26)-(3.28) and (3.29) gives for $d_{1} \geqslant d_{1}(\aleph)$

$$
\mathbb{P}\left(Z_{1}(1)=Z_{2}(1), B^{2}\right) \geqslant \tilde{p}=\tilde{p}\left(p, \aleph, R_{1}\right)>0 .
$$

Taking into account the definition of $\phi$ and choosing $d_{0}=2 d_{1}$, it follows that (3.19) holds.

\section{Appendix A. Proof of Lemma 1.2}

Let $\left(Y_{i}\right)_{i}$ be a coupling of $\left(\mu_{i}\right)_{i}$. Let $\Gamma$ be a measurable set. There exists $\left(\Gamma_{i}\right)_{i}$ such that

$$
\Gamma=\bigcup_{i} \Gamma_{i}, \quad \bigcap_{i} \Gamma_{i}=\emptyset, \quad\left(\mu_{2}-\mu_{1}\right)^{+}\left(\Gamma_{2}\right)=0, \quad\left(\mu_{1}-\mu_{2}\right)^{+}\left(\Gamma_{1}\right)=0 .
$$

It follows from $a \wedge b=a-(a-b)^{+}$and $\left(\mu_{1}-\mu_{2}\right)^{+}\left(\Gamma_{1}\right)=0$ that

$$
\left(\mu_{1} \wedge \mu_{2}\right)\left(\Gamma_{1}\right)=\mu_{1}\left(\Gamma_{1}\right)-\left(\mu_{1}-\mu_{2}\right)^{+}\left(\Gamma_{1}\right)=\mu_{1}\left(\Gamma_{1}\right)=\mathbb{P}\left(Y_{1} \in \Gamma_{1}\right) .
$$


Symmetrical, we obtain $\left(\mu_{1} \wedge \mu_{2}\right)\left(\Gamma_{2}\right)=\mathbb{P}\left(Y_{2} \in \Gamma_{2}\right)$.

Thus, it follows from $\Gamma=\bigcup_{i} \Gamma_{i}$ and $\bigcap_{i} \Gamma_{i}=\emptyset$ that

$$
\left(\mu_{1} \wedge \mu_{2}\right)(\Gamma)=\mathbb{P}\left(Y_{1} \in \Gamma_{1}\right)+\mathbb{P}\left(Y_{2} \in \Gamma_{2}\right) \geqslant \sum_{i=1}^{2} \mathbb{P}\left(Y_{1}=Y_{2}, Y_{1} \in \Gamma_{i}\right)
$$

Since $\Gamma=\bigcup_{i} \Gamma_{i}$ and $\bigcap_{i} \Gamma_{i}=\emptyset$, then

$$
\left(\mu_{1} \wedge \mu_{2}\right)(\Gamma) \geqslant \mathbb{P}\left(Y_{1}=Y_{2}, Y_{1} \in \Gamma\right) .
$$

Then it follows from $\left\|\mu_{1}-\mu_{2}\right\|_{\mathrm{var}}=1-\left(\mu_{1} \wedge \mu_{2}\right)(E)$ that

$$
\left\|\mu_{1}-\mu_{2}\right\|_{\mathrm{var}} \leqslant \mathbb{P}\left(Y_{1} \neq Y_{2}\right) .
$$

We have equality only if (A.1) appears for $\Gamma=E$, which is true only if (A.1) appears for any $\Gamma$. For any measure $\mu$ on $(E, \mathcal{E})$, we denote by $\mu$ the measure on $(E, \mathcal{E}) \otimes(E, \mathcal{E})$ define by

$$
\mu(\mathbf{A})=\mu(\{a \in E \mid(a, a) \in \mathbf{A}\}) .
$$

If $\mu_{1}=\mu_{2}$, we set $\mathbb{P}=\mu_{\mathbf{1}}$. Else we set

$$
\mathbb{P}=\mu_{1} \wedge \mu_{2}+\frac{1}{\left\|\mu_{2}-\mu_{1}\right\|_{\mathrm{var}}}\left(\mu_{1}-\mu_{2}\right)^{+} \otimes\left(\mu_{2}-\mu_{1}\right)^{+} .
$$

Noticing that $a=a \wedge b+(a-b)^{+}$and using $\left\|\mu_{1}-\mu_{2}\right\|_{\mathrm{var}}=\left(\mu_{1}-\mu_{2}\right)^{+}(E)$, we obtain that $\mathbb{P}(\cdot \times E)=\mu_{1} \wedge \mu_{2}+$ $\left(\mu_{1}-\mu_{2}\right)^{+}=\mu_{1}$ and $\mathbb{P}(E \times \cdot)=\mu_{2}$. Thus if we denote by $\left(Y_{i}\right)_{i}$ the projectors, we obtain that $\left(Y_{i}\right)_{i}$ is a coupling of $\left(\mu_{i}\right)_{i}$. Moreover,

$$
\mathbb{P}\left(Y_{1}=Y_{2}, Y_{1} \in A\right)=\left(\mu_{1} \wedge \mu_{2}\right)(A) .
$$

So it is the desired maximal coupling

Remark A.1. Moreover, in all this article, we admit that the maximal coupling $\left(Y_{i}\left(u_{0}^{i}\right)\right)_{i}$ could be chosen such that $\left(Y_{i}\left(u_{0}^{1}, u_{0}^{2}\right)\right)_{i}$ depend measurably on the initials conditions $\left(u_{0}^{i}\right)_{i}$. The idea is the following. Since we only work in nice spaces, we can consider that we are working on the real line. It can be seen that the laws we use depend measurably on $\left(u_{0}^{i}\right)_{i}$ and then the law define by (A.2) will do it too. Then its repartition function $F_{\left(u_{0}^{1}, u_{0}^{2}\right)}$ is measurable too and finally the pseudo-inverse of the repartition function $F_{\left(u_{0}^{1}, u_{0}^{2}\right)}^{-1}$ is measurable with respect to $\left(u_{0}^{1}, u_{0}^{2}\right)$. We consider $\left([0,1], \mathcal{B}_{[0,1]}, \lambda\right)$, where $\lambda$ is the Lebesgue measure and we set $Y_{i}\left(u_{0}^{1}, u_{0}^{2}, \omega\right)=F_{\left(u_{0}^{1}, u_{0}^{2}\right)}^{-1}(\omega)$. Then $\left(Y_{i}\right)_{i}$ is measurable with respect to $\left(u_{0}^{1}, u_{0}^{2}, \omega\right)$ and for every $\left(u_{0}^{1}, u_{0}^{2}\right)$, it is a coupling of $\left(\mu_{i}\left(u_{0}^{i}\right)\right)_{i}$. For a proof see [13].

\section{Appendix B. Proof of Proposition 1.7}

We set

$$
\Omega=E^{2}, \quad \mathcal{F}=\mathcal{B}\left(E^{2}\right),
$$

and $V_{i}$ the $i$ th projector on $\Omega$ :

$$
V_{i}\left(v_{1}, v_{2}\right)=v_{i}, \quad i=1,2 .
$$

Let $\left(U_{1}, U_{2}\right)$ be a coupling of $\left(\mu_{1}, \mu_{2}\right)$.

In order to establish Proposition 1.7, we build a probability measure $Q$ on $(\Omega, \mathcal{F})$ such that

$$
\left\{\begin{array}{l}
(\alpha) Q(\cdot \times E)=\mu_{1}, \quad Q(E \times \cdot)=\mu_{2}, \\
(\beta) Q\left(f_{0}\left(V_{1}\right)=f_{0}\left(V_{2}\right)\right) \geqslant\left(v_{1} \wedge v_{2}\right)(E) .
\end{array}\right.
$$

Then $\left(V_{1}, V_{2}\right)$ seen as a couple of random variables defined on $(\Omega, \mathcal{F}, Q)$ is a coupling of $\left(\mu_{1}, \mu_{2}\right)$ such that $\left(f\left(V_{1}\right), f\left(V_{2}\right)\right)$ is a maximal coupling of $\left(v_{1}, v_{2}\right)$. 
Recall that

$$
v_{i}=v_{1} \wedge v_{2}+\left((-1)^{i}\left(v_{1}-v_{2}\right)\right)^{+}, \quad i=1,2,
$$

and that since $E, F$ are polish spaces, then there exists a version of $\mathbb{P}\left(U_{i} \in A \mid f_{0}\left(U_{i}\right)=x\right)$ which is measurable for any $A \in \mathcal{B}(E)$ and which is probability measure for any $x \in F$. Moreover

$$
\mu_{i}(A)=\int_{F} \mathbb{P}\left(U_{i} \in A \mid f_{0}\left(U_{i}\right)=x\right) v_{i}(\mathrm{~d} x), \quad i=1,2 .
$$

Combining (B.2) and (B.3), we obtain

$$
\mu_{i}=\mu_{i}^{s}+\mu_{i}^{r}, \quad i=1,2,
$$

where

$$
\begin{aligned}
& \mu_{i}^{s}(A)=\int_{F} \mathbb{P}\left(U_{i} \in A \mid f_{0}\left(U_{i}\right)=x\right)\left(v_{1} \wedge v_{2}\right)(\mathrm{d} x), \quad i=1,2, \\
& \mu_{i}^{r}(A)=\int_{F} \mathbb{P}\left(U_{i} \in A \mid f_{0}\left(U_{i}\right)=x\right)\left((-1)^{i}\left(v_{1}-v_{2}\right)\right)^{+}(\mathrm{d} x), \quad i=1,2 .
\end{aligned}
$$

\section{Remark that}

$$
\left\{\begin{array}{l}
\mu_{i}^{s}, \mu_{i}^{r} \geqslant 0, \quad i=1,2, \\
\mu_{i}^{s}(E)=\left(v_{1} \wedge v_{2}\right)(E), \\
\mu_{i}^{r}(E)=\left\|v_{1}-v_{2}\right\|_{\mathrm{var}} .
\end{array}\right.
$$

Taking into account (B.4) and (B.5), we can write problem (B.1) in the form

$$
\left\{\begin{array}{l}
\text { Find } r, s \text { two positive measures on }(\Omega, \\
\text { (i) } s(\cdot \times E)=\mu_{1}^{s}, \quad s(E \times \cdot)=\mu_{2}^{s}, \\
\text { (ii) } r(\cdot \times E)=\mu_{1}^{r}, \quad r(E \times \cdot)=\mu_{2}^{r}, \\
\text { (iii) } s\left(f_{0}\left(V_{1}\right) \neq f_{0}\left(V_{2}\right)\right)=0 .
\end{array}\right.
$$

Once (B.6) is true, we can set

$$
Q=r+s .
$$

Then (B.1)( $\alpha$ ) is an obvious consequence of (B.4). Furthermore, since $r \geqslant 0$, then (B.6)(iii), (B.6)(i) and (B.5) gives

$$
Q\left(f_{0}\left(V_{1}\right)=f_{0}\left(V_{2}\right)\right) \geqslant s\left(f_{0}\left(V_{1}\right)=f_{0}\left(V_{2}\right)\right)=s(\Omega)=\mu_{i}^{s}(E)=\left(v_{1} \wedge v_{2}\right)(E) .
$$

Now we build $r$ by setting

$$
r=\frac{1}{\left\|v_{1}-v_{2}\right\|_{\mathrm{var}}} \mu_{1}^{r} \times \mu_{2}^{r} .
$$

Notice that $r \geqslant 0$ and (B.6)(ii) are obvious consequence of (B.5).

Now we build $s$ by setting

$$
s(A \times B)=\int_{F} \mathbb{P}\left(U_{1} \in A \mid f_{0}\left(U_{1}\right)=x\right) \times \mathbb{P}\left(U_{2} \in B \mid f_{0}\left(U_{2}\right)=x\right)\left(v_{1} \wedge v_{2}\right)(\mathrm{d} x) .
$$

Notice that (B.6)(i) and (B.6)(iii) are obvious.

\section{References}

[1] M. Barton-Smith, Invariant measure for the stochastic Ginzburg Landau equation, NoDEA Nonlinear Differential Equations Appl. 11 (1) (2004) 29-52.

[2] P. Bebouche, A. Jüngel, Inviscid limits of the Complex Ginzburg-Landau equation, Comm. Math. Phys. 214 (2000) $201-226$. 
[3] J. Bricmont, A. Kupiainen, R. Lefevere, Exponential mixing for the 2D stochastic Navier-Stokes dynamics, Comm. Math. Phys. 230 (1) (2002) 87-132.

[4] G. Da Prato, J. Zabczyk, Stochastic equations in infinite dimensions, in: Encyclopedia Math. Appl., Cambridge University Press, 1992.

[5] A. de Bouard, A. Debussche, A stochastic non-linear Schrödinger equation with multiplicative noise, Comm. Math. Phys. 205 (1999) 161-181.

[6] A. de Bouard, A. Debussche, The stochastic non-linear Schrödinger equation in $H^{1}$, Stochastic Anal. Appl. 21 (1) (2003) 97-126.

[7] W. E, J.C. Mattingly, Y.G. Sinai, Gibbsian dynamics and ergodicity for the stochastically forced Navier-Stokes equation, Comm. Math. Phys. 224 (2001) 83-106.

[8] V. Ginzburg, L. Landau, On the theorie of superconductivity, Zh. Eksp. Fiz. 20 (1950) 1064; English transl. in: Ter Haar (Ed.), Men of Physics: L.D. Landau, vol. I, Pergamon Press, New York, 1965, pp. 546-568.

[9] M. Hairer, Exponential mixing properties of stochastic PDEs through asymptotic coupling, Probab. Theory Related Fields 124 (3) (2002) 345-380.

[10] G. Huber, P. Alstrom, Universal decay of vortex density in two dimensions, Physica A 195 (1993) 448-456.

[11] S. Kuksin, On exponential convergence to a stationary measure for nonlinear PDEs, in: The M.I. Viishik Moscow PDE seminar, in: Amer. Math. Soc. Transl. Ser. (2), vol. 206, Amer. Math. Soc., 2002.

[12] S. Kuksin, A. Shirikyan, Stochastic dissipative PDE's and Gibbs measures, Comm. Math. Phys. 213 (2000) 291-330.

[13] S. Kuksin, A. Shirikyan, A coupling approach to randomly forced PDE's I, Comm. Math. Phys. 221 (2001) 351-366.

[14] S. Kuksin, A. Piatnitski, A. Shirikyan, A coupling approach to randomly forced PDE's II, Comm. Math. Phys. 230 (1) (2002) $81-85$.

[15] S. Kuksin, A. Shirikyan, Coupling approach to white-forced nonlinear PDEs, J. Math. Pures Appl. 1 (2002) 567-602.

[16] S. Kuksin, A. Shirikyan, Randomly forced CGL equation: Stationary measure and the inviscid limit, J. Phys. A 37 (12) (2004) $3805-3822$.

[17] J. Mattingly, Exponential convergence for the stochastically forced Navier-Stokes equations and other partially dissipative dynamics, Comm. Math. Phys. 230 (2002) 421-462.

[18] J. Mattingly, On recent progress for the stochastic Navier-Stokes equations, in: Journées Équations aux Dérivées Partielles, Exp. No XI, vol. 52, Univ. Nantes, Nantes, 2003.

[19] A. Newel, J. Whitehead, Finite bandwidth, finite amplitude convection, J. Fluid Mech. 38 (1969) 279-303.

[20] A. Newel, J. Whitehead, Review of the finite bandwidth concept, in: H. Leipholz (Ed.), Proceedings of the Internat. Union of Theor. and Appl. Math., Springer, Berlin, 1971, pp. 284-289.

[21] C. Odasso, Propriétés ergodiques de l'équation de Ginzburg-Landau complexe bruitée, Mémoire de DEA, 2003.

[22] A. Shirikyan, Exponential mixing for 2D Navier-Stokes equation perturbed by an unbounded noise, J. Math. Fluid Mech. 6 (2) (2004) 169193. 